\title{
Is there any evidence that ionized outflows quench star formation in type 1 quasars at $z<1$ ?
}

\author{
B. Balmaverde ${ }^{1,2}$, A. Marconi ${ }^{1,2}$, M. Brusa ${ }^{3,4}$, S. Carniani ${ }^{6}$, G. Cresci ${ }^{1}$, E. Lusso ${ }^{1}$, R. Maiolino ${ }^{5,6}$, \\ F. Mannucci ${ }^{1}$, and T. Nagao ${ }^{7}$
}

\author{
1 Dipartimento di Fisica e Astronomia, Università di Firenze, via G. Sansone 1, 50019 Sesto Fiorentino (Firenze), Italy \\ 2 INAF-Osservatorio Astrofisico di Arcetri, Largo Enrico Fermi 5, 50125 Firenze, Italy \\ 3 Dipartimento di Fisica e Astronomia, Universitàă di Bologna, viale Berti Pichat 6/2, 40127 Bologna, Italy \\ ${ }^{4}$ INAF-Osservatorio Astronomico di Bologna, via Ranzani 1, 40127 Bologna, Italy \\ ${ }^{5}$ Cavendish Laboratory, University of Cambridge, 19 J. J. Thomson Ave, Cambridge CB3 OHE, UK \\ ${ }^{6}$ Kavli Institute for Cosmology, University of Cambridge, Madingley Road, Cambridge CB3 0HA, UK \\ 7 Research Center for Space and Cosmic Evolution, Ehime University, Bunkyo-cho 2-5, 790-8577 Matsuyama, Japan
}

Received 8 June 2015 / Accepted 11 September 2015

\section{ABSTRACT}

\begin{abstract}
Aims. The aim of this paper is to test the basic model of negative active galactic nuclei (AGN) feedback. According to this model, once the central black hole accretes at the Eddington limit and reaches a certain critical mass, AGN driven outflows blow out gas, suppressing star formation in the host galaxy and self-regulating black hole growth.

Methods. We consider a sample of 224 quasars selected from the Sloan Digital Sky Survey (SDSS) at $z<1$ observed in the infrared band by the Herschel Space Observatory in point source photometry mode. We evaluate the star formation rate in relation to several outflow signatures traced by the [O III] $\lambda 4959,5007$ and [O II] $\lambda 3726,3729$ emission lines in about half of the sample with high quality spectra.

Results. Most of the quasars show asymmetric and broad wings in [O III], which we interpret as outflow signatures. We separate the quasars in two groups, "weakly" and "strongly" outflowing, using three different criteria. When we compare the mean star formation rate in five redshift bins in the two groups, we find that the star formation rate (SFR) are comparable or slightly larger in the strongly outflowing quasars. We estimate the stellar mass from spectral energy distribution (SED) fitting and the quasars are distributed along the star formation main sequence, although with a large scatter. The scatter from this relation is uncorrelated with respect to the kinematic properties of the outflow. Moreover, for quasars dominated in the infrared by starburst or by AGN emission, we do not find any correlation between the star formation rate and the velocity of the outflow, a trend previously reported in the literature for pure starburst galaxies.

Conclusions. We conclude that the basic AGN negative feedback scenario seems not to agree with our results. Although we use a large sample of quasars, we did not find any evidence that the star formation rate is suppressed in the presence of AGN driven outflows on large scale. A possibility is that feedback is effective over much longer timescales than those of single episodes of quasar activity.
\end{abstract}

Key words. quasars: general - galaxies: active - galaxies: star formation

\section{Introduction}

An important discovery in the last 15 years is that the black hole mass at the centre of the galaxies is a constant fraction of the mass of the stellar bulge (Ferrarese \& Merritt 2000; Gebhardt et al. 2000). From that moment it has been clear that the evolution of galaxies is closely connected with the growth of the central black hole and that the influence of the black hole on the host galaxy cannot be ignored.

In particular AGN feedback (i.e. the energy released by the active galactic nucleus into the interstellar medium) is invoked to solve many astrophysical problems. For example, the very sharp cut-off seen at the bright end of the galaxies luminosity function cannot be reproduced without invoking a feedback mechanism. In other words, super-winds may be responsible for the lack of bright galaxies that are not observed in reality (Benson et al. 2003) and for the red, dead elliptical galaxies we observe today. Another crucial problem that requires AGN feedback is the so-called cooling flow problem at the centre of galaxy clusters, which requires a source of heating able to balance or quench cooling (Fabian 2012, and references therein).

A wide variety of quenching mechanisms have been proposed to self-regulate the growth of the galaxy (i.e. the star formation rate) and the black hole mass accretion rate (e.g. Di Matteo et al. 2005; Hopkins et al. 2006; Croton 2006; Martig et al. 2009). Generally, the quenching is identified with a triggering event, for example a major merger, that channels a large amount of gas into the central region in a short period of time, activating both star formation (SF) and quasar activity. The resulting AGN feedback rapidly exhausts or sweeps away the gas from the galaxy. Theoretical models (e.g. Hopkins et al. 2006; Granato et al. 2004; Di Matteo et al. 2005) have proposed that feedback from AGNs is responsible for the regulation of SF, transforming a starburst galaxy into a red elliptical galaxy.

In a considerable fraction of AGNs there are clear signatures of outflows likely able to remove significant amounts of cold gas from the galaxy. AGN-driven outflows with velocities of $\sim 1000 \mathrm{~km} \mathrm{~s}^{-1}$ have been identified in ultra luminous infra red 
galaxies (ULIRGs), e.g. Cicone et al. (2014); Rupke \& Veilleux (2013), and fast outflows are seen in broad absorption line QSOs displaying broad UV absorption lines with widths of several thousand $\mathrm{km} \mathrm{s}^{-1}$ (Turnshek et al. 1988). The highly ionized Fe absorption lines blueshifted by $0.05-0.3 \mathrm{c}$ observed in the X-ray bands (e.g. Tombesi et al. 2010) have been found associated with the much slower $\left(\sim 1000 \mathrm{~km} \mathrm{~s}^{-1}\right)$ outflow components seen in the molecular gas (e.g. Feruglio et al. 2015; Tombesi et al. 2015). On the galactic scale, integral field spectroscopic observations have revealed the presence of ionized gas outflows with velocites up to $1000 \mathrm{~km} \mathrm{~s}^{-1}$ in low and high redshift galaxies (Alexander et al. 2010; Harrison et al. 2012, 2014; Cano-Díaz et al. 2012).

However, studies exploring the role of AGNs with respect to star formation have led to ambiguous and contrasting results, with evidence for both negative and positive impact of AGN outflows on star formation. After many years of extensive research, conclusive proof that AGN feedback is able to halt galaxy-wide star formation is still lacking. Moreover, many studies show that the opposite may actually be true. The squeezing and compression of cold gas induced by accretion related winds could cause local density fluctuations leading to star formation (Ishibashi \& Fabian 2012). Theoretical models able to explain the connection between the central engine and the host galaxy properties with secular evolution without invoking AGN feedback have been developed (e.g. Jahnke \& Macciò 2011; Ciotti \& Ostriker 2007). Finally, even if it is well known that the AGN driven outflows are common in both radio loud and radio quiet quasars, it is not obvious if there is an efficient coupling mechanism between the AGN and the ISM (e.g. Bicknell et al. 2000; Kalfountzou et al. 2012).

In this paper we focus on ionized gas outflows traced by the optical [O III] and [O II] lines. Since the forbidden line emission is isotropic and self-absorption in narrow lines is negligible, the blue wings are generally interpreted as the result of outflowing gas. The [OIII] line is typical for AGNs and originates from the ionized narrow-line region (NLR) gas surrounding the accreting super massive black hole in the centre of the galaxy (see Osterbrock 1989). Mullaney et al. (2013) constructed average [O III] $\lambda 5007$ profiles derived from stacking SDSS spectra of type 1 AGNs and found profiles displaying prominent blue wings shifted with respect to the narrow components. Similar results are found by Zakamska \& Greene (2014) who analysed the SDSS spectra of obscured luminous quasars. The [O III] $\lambda 5007$ emission line typically shows blueshifts and blue excess, likely signatures that the NLR is undergoing an outflow.

The question we address in this paper is whether SMBH outflows and feedback have the ability to regulate the starburst on a galaxy-wide scale as predicted by the negative feedback scenario. To this end, we compare the kinematic of the ionized gas outflows derived from the [O III] line profile to the star formation rate of the host galaxies estimated through the fit of photo-metric points in the infrared. Since the two processes likely act on different timescales (e.g. Stanley et al. 2015, and references therein) we need a large sample of luminous powerful quasars in order to recover the signature that AGN outflows affect the star formation rate of the host galaxy. Direct Herschel FIR measurements of individual galaxies is a unique tool used to properly estimate the ongoing star formation rate, especially in low luminosity IR galaxies, that in large sky surveys would not be detected. Therefore, we selected a large sample of quasars at redshift $\lesssim 1$ (to cover the [O III] emission line wavelength in SDSS optical spectra) observed by the infrared telescope Herschel in photometric point source mode.

The paper is organized as follows. In Sect. 2 we define the sample of quasars, and in Sect. 3 we present the analysis of the SDSS spectra and of the Hershel data. The main results and the comparison between the outflow properties, the star formation rate (SFR) and AGN properties, are presented in Sects. 4 and 5. In Sect. 6 we provide a discussion, and in Sect. 7 our summary and conclusions.

In this paper we assume the following cosmology parameters: $\Omega_{\mathrm{m}}=0.3, \Lambda=0.7$ and $H_{0}=70 \mathrm{~km} \mathrm{~s}^{-1} \mathrm{Mpc}^{-1}$.

\section{The sample}

The AGN parent sample includes bona fide quasars extracted from the Sloan Digital Sky Survey (SDSS) archive or the SDSS quasar catalogue that have high resolution photometric images from the infrared satellite Herschel. from

First of all, we selected a list of reliable quasars candidates

- the fifth edition of the spectroscopic quasar catalogue of Schneider et al. (2010) based on the SDSS seventh data release (DR7Q). This catalogue contains 19552 quasars at redshifts of less than 0.8 (this redshift limit ensures that [O III] is in the observed frame);

- the quasar catalogue of Pâris et al. (2012) based on the SDSS ninth data release (DR9Q). There are 23964 quasars at redshifts of less than 1 ;

- the SDSS archive. We searched for objects classified as "QSO" in the latest SDSS data release (DR10) not present in the two quasar catalogues with redshifts of less than 1 and a median signal-to-noise ratio greater than 5 (35 830 quasars).

These subsamples are selected according to several different criteria. The DR7Q catalogue contains quasars mostly at $z<2$ (see Shen et al. 2011, for their properties) selected with a complex algorithm described in Richards et al. (2002). The DR9Q quasar catalogue contains mostly newly discovered quasars, but also some DR7 quasars (about 16420) that were re-observed during the Baryon Oscillation Spectroscopic Survey (BOSS, Schlegel et al. 2007). All quasars have absolute $i$-band magnitudes brighter than -22 , contain at least one broad emission line (with $F W H M>500 \mathrm{~km} \mathrm{~s}^{-1}$ ) or - if they do not - have interesting/complex absorption features, and have highly reliable redshifts. The threshold in absolute magnitude was relaxed for DR9Q $\left(M_{i}>-20.5\right)$ because BOSS were targeting mostly $z>2.15$ quasars.

We then searched in the Herschel archive for PACS and/or SPIRE images covering a portion of a circle of five arcmin of radius around the quasars position for all the $\sim 79000$ quasars. We searched for the nearest source within a circular region of $20^{\prime \prime}$ centred on SDSS quasar position. We found 224 quasars observed by Herschel in point source photometry mode providing the best instrumental sensitivity and resolution available (114 from DR7Q, 92 from DR9Q, and 18 from DR10 data archive). In Fig. 1 we show the distribution of the redshift values for the quasars in our sample.

\section{Data analysis}

\subsection{Herschel photometry}

The Herschel Space Observatory is equipped with a $3.5 \mathrm{~m}$ telescope that has performed photometry and spectroscopy in 


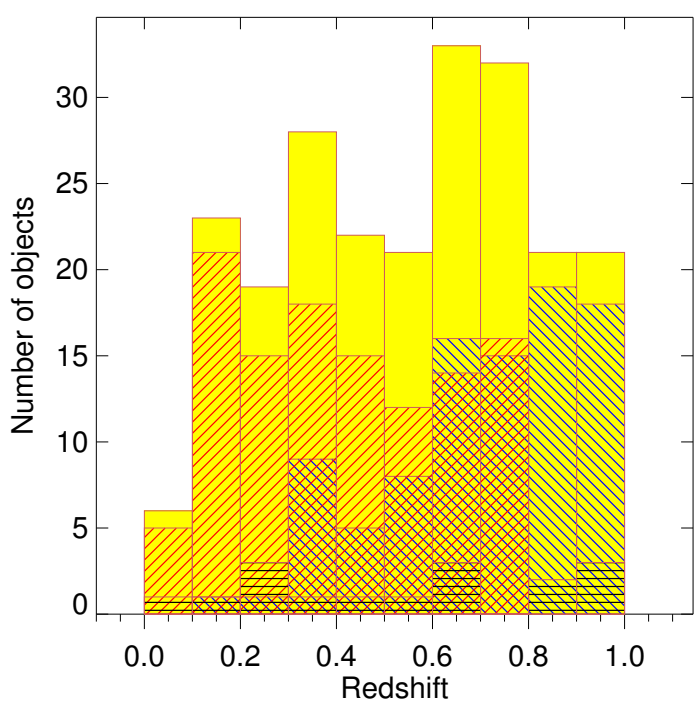

Fig. 1. Histogram of the redshift distribution for the quasars in our sample. The red, blue, and black hatched areas identify quasars from the DR7Q, DR9Q, and SDSS archives, respectively. The yellow area denotes the sum of the three subsamples.
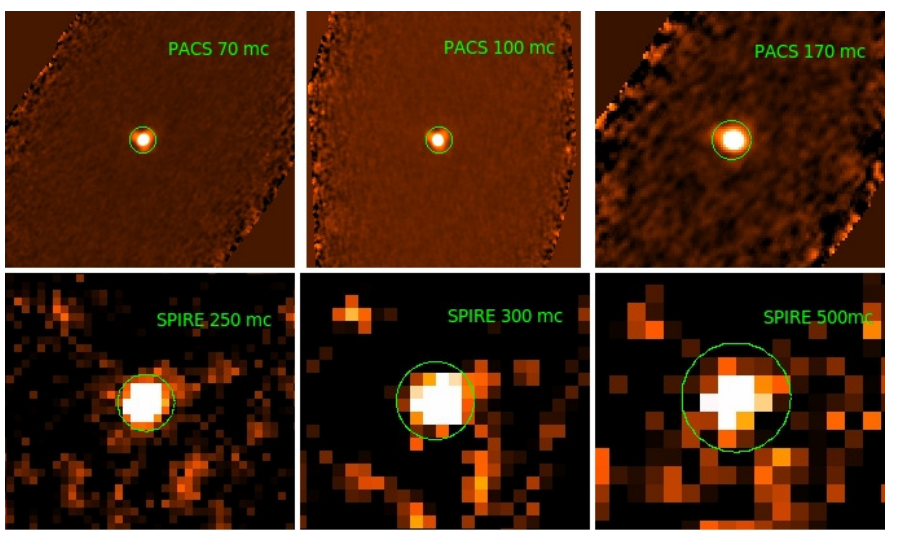

Fig. 2. Herschel PACS and SPIRE images in different bands for a quasar from our sample. The green circle is centred on the source and represents the extraction region.

approximately the 55-671 $\mu \mathrm{m}$ range, mapping the "warm" dust heated by young stars. There are two photometric instruments, the Photodetecting Array Camera and Spectrometer (PACS), which observes in three colours (blue 60-85 $\mu \mathrm{m}$, green $85-130 \mu \mathrm{m}$, and red $130-210 \mu \mathrm{m})$, and the Spectral and Photometric Imaging Receiver (SPIRE), which observes at 250, 350, and $500 \mu \mathrm{m}$ (Poglitsch et al. 2010; Griffin et al. 2010).

We perform aperture photometry on PACS and SPIRE pipeline-processed images using the annularSkySperturePhotometry task in HIPE version 12.0. We adopt a circular target aperture centred on the position of the target of radius $10^{\prime \prime}$ and $15^{\prime \prime}$ for blue/green and red PACS images, respectively, and $22^{\prime \prime}, 30^{\prime \prime}$, and $42^{\prime \prime}$ for the three SPIRE bands (see Fig. 2). The background annulus is chosen between $20^{\prime \prime}$ and $40^{\prime \prime}$ for PACS blue/green bands, between $25^{\prime \prime}$ and $45^{\prime \prime}$ for PACS red band, and between $65^{\prime \prime}$ and 95" for SPIRE images (see PACS and SPIRE data reduction guide ${ }^{1}$ for details on the PSF shape and the suggested photometry aperture). The default algorithm for the sky estimation is the same used in the IDL daophot package, which eliminate the outlier pixels, i.e. values with a low probability given a Gaussian with specified average and sigma. We define

1 http://herschel.esac.esa.int/hcss-doc-14.0/ the luminosity errors $\sigma_{\text {src }}$ as the square root of the quadratic sum of two terms, $\sqrt{N_{\text {src_pix }}} \sigma_{\text {sky }}$, and the error on the mean of sky $\sqrt{N_{\text {src_pix }}} \frac{\sigma_{\text {sky }}}{\sqrt{N_{\text {sky }}}}$ (however the second term is irrelevant because the sky is measured on a much larger area with respect to the extraction region); $\sigma_{\text {src }}$ is therefore given by the uncertainty of the background times the square root of the number of pixels in the extraction region, $N_{\text {src_pix }}$.

If we do not reach a signal-to-noise ratio $(\mathrm{S} / \mathrm{N}$, defined as the ratio of the flux density $\mathrm{S}$ over the uncertainty error $\sigma_{\text {src }}$ ) of at least a factor 3, we estimate an upper limit for the flux density at $2 \sigma$ confidence level as $2 \times \sigma_{\text {src }} \times \sqrt{N_{\text {src_pix }}}$ (in this case $\sigma_{\text {src }}$ is the standard deviation of the flux density mean values in the extraction region). Finally we applied aperture correction using the task photApertureCorrectionPointSource.

The sensitivity of the Herschel instruments depends on many factors, but to a first order in all the observing modes it scales with the inverse of the square root of the on-source observation time. The typical sensitivity for a $5 \sigma$ detection in one hour of observation is of the order of $5 \mathrm{mJy}$ for PACS in the $70 / 100 \mu \mathrm{m}$ band and $10 \mathrm{mJy}$ for SPIRE in the $160 \mu \mathrm{m}$ band $^{2}$. In Fig. 3, we compare the PACS AND SPIRE fluxes in all the bands with this approximate sensitivity threshold. The median exposure time for our data is comparable, and all our upper limits are located below the threshold for detectability in one hour of observation and the detections (with very few exceptions) are located above these lines.

\subsection{Ancillary data}

We build the spectral energy distribution (SED) of the 224 quasars by adding photometric points from $\sim 0.3$ to $22 \mu \mathrm{m}$ band to the Herschel data, taking advantage of optical and infrared all-sky surveys (SDSS, 2MASS, WISE).

In the optical range, SDSS photometric system comprises five colour bands $(u, g, r, i, z)$ from about 0.3 to $0.9 \mu \mathrm{m}$. We choose the quantity psfMag, for which the total flux is determined by fitting a PSF model to the object.

The Two Micron Sky Survey mapped the entire sky in the $J(1.24 \mu \mathrm{m}), H(1.66 \mu \mathrm{m})$, and $K(2.16 \mu \mathrm{m})$ near-infrared bands with a pixel size of $2.0^{\prime \prime}$. We considered sources in 2MASS from the All Sky Point Source Catalogue and the Reject Table within $2^{\prime \prime}$ matching radius. If the QSO is not detected, we downloaded the image of the field of view around the quasars position from the 2MASS archive, and we used the IDL routine aper (adapted from DAOPHOT) to estimate the limiting magnitude at the position of the quasar. In detail we derive the magnitude from a 4 " radius region centred on the expected source position and we estimate the sky background in an annular region with inner radius of $14^{\prime \prime}$ and outer radius of $20^{\prime \prime}$. To decide whether the source is detected in the image, we repeat the measurement in 1000 circular regions randomly selected around 100" from the source. We then build the distribution of all 1000 flux values (mostly from empty regions of the sky) and if the flux of the source is higher than the 95th percentile of this distribution, we consider this measurement a detection, otherwise the 95th percentile as a $2 \sigma$ upper limit.

The Wide-field Infrared Survey Explorer (WISE) is an infrared space telescope that performed an all-sky astronomical survey in the $W 1(3.4 \mu \mathrm{m}), W 2(4.6 \mu \mathrm{m}), W 3(12 \mu \mathrm{m})$, and $W 4(22 \mu \mathrm{m})$ bands. All 224 quasars in our sample are

\footnotetext{
2 http://herschel.esac.esa.int/Docs/Herschel/html/ ch03s02.html
} 

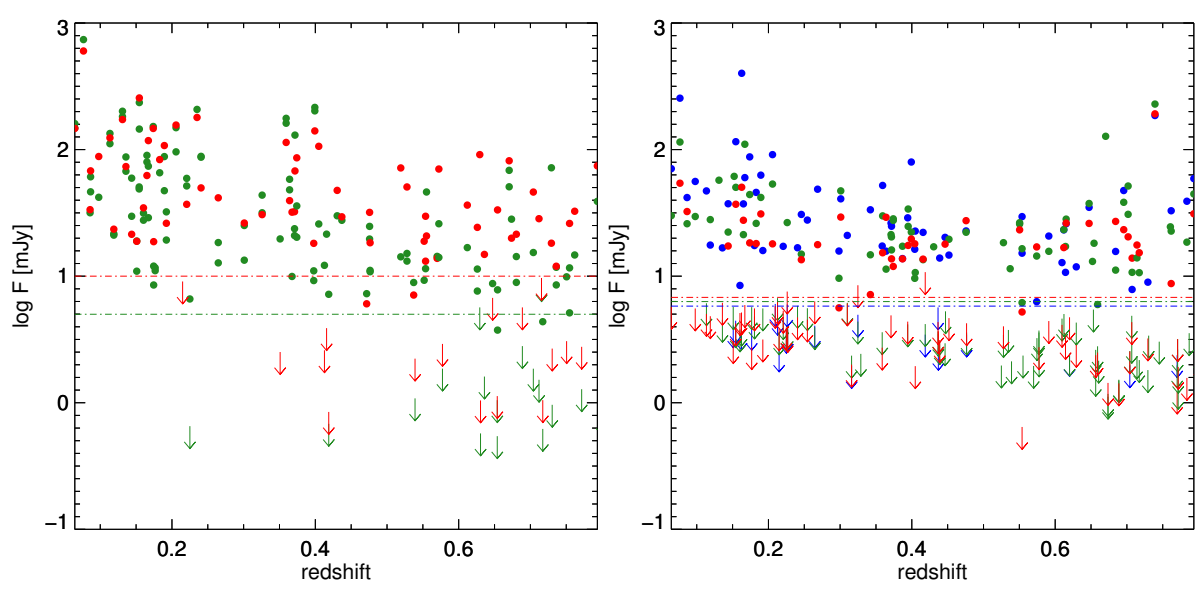

Fig. 3. Left panel: observed source fluxes in the PACS bands at $70 / 100 \mu \mathrm{m}$ and $160 \mu \mathrm{m}$ (green and red points, respectively), compared with the detector sensitivity threshold at $5 \sigma$ for one hour of observation (shown as dash-dotted green and red lines). Right panel: same figure for the SPIRE band at $250 \mu \mathrm{m}, 350 \mu \mathrm{m}, 500 \mu \mathrm{m}$ (blue, green, and red points, respectively). The three dashdotted lines are the detector sensitivity thresholds in the three bands at $5 \sigma$ for one-hour observations. detected in at least one WISE band and are present in the WISE All-Sky Source Catalog. When the flux measurement has a signal-to-noise ratio less than 2 , the magnitudes are replaced with the $2 \sigma$ brightness upper limit in magnitude units.

To characterize the radio properties of our quasars, we follow the same approach adopted by Shen et al. (2011) to include the radio loudness parameter in the SDSS-DR7 catalogue quasars. The standard radio loudness parameter is defined as the ratio between the flux density $\left(f_{v}\right)$ at rest-frame $6 \mathrm{~cm}$ and $2500 \AA(R=$ $f_{6 \mathrm{~cm}} / f_{2500 \AA}$, e.g. Jiang et al. 2007) ${ }^{3}$. About $25 \%$ of the quasars (i.e., 50/224) have been detected in the FIRST catalogue and $34 / 224$ have a radio loudness parameter greater than $10(\sim 15 \%$ of our quasars are radio loud).

\subsection{SED fitting}

The infrared emission is dominated by the black-body emission of dust at different temperatures, heated by the optical and UV emission of the AGN and of young and old stars. A major difficulty in deriving the star formation rate from infrared luminosity is to disentangle contributions from different sources of heating. Typically the dusty torus can reach dust sublimation temperatures of the order of $1700 \mathrm{~K}$ and the peak of the emission is at shorter wavelength (less than $100 \mu \mathrm{m}$ ) than that produced by warm dust around young stars. In star-forming galaxies there is a considerable overlap around 3-20 $\mu \mathrm{m}$ where the polycyclic aromatic hydrocarbon (PAH) emission may dominate the IR emission. However, this is not problematic because PAH features are almost absent in AGNs since molecules should be destroyed by the extreme optical-UV and soft X-ray radiation in AGN (Roche et al. 1991; Siebenmorgen et al. 2004). At shorter wavelengths (around 3-4 $\mu \mathrm{m}$ ), the emission is dominated by older stars and luminosities are thus strongly correlated with stellar mass (Meidt et al. 2012).

In the literature there are many infrared templates for modelling galaxy infrared spectral energy distribution. Some of these models are multi-dimensional, in the sense that there is a grid of parameters from which one can derive many physical characteristics of the system (e.g. Silva et al. 1998; Draine \& Li 2007). Other are one-dimensional, i.e. they rely on a single parameter

\footnotetext{
3 We match our sample with the VLA FIRST Survey at $20 \mathrm{~cm}$ with a searching radius of $30^{\prime \prime}$ and we derive the rest-frame $6 \mathrm{~cm}$ flux density from the FIRST integrated flux density at $20 \mathrm{~cm}$ assuming a power-law slope of $\alpha_{v}=-0.5$. The rest-frame $2500 \AA$ flux density is determined from the fit to the continuum around $5100 \AA$ fitted with a power-law shape $\left(f_{\lambda}=A \lambda^{\alpha}\right)$.
}

to characterize the shape of the model (e.g. Chary \& Elbaz 2001; and Rieke et al. 2009).

The measurements in low IR-luminosity quasars is of fundamental importance in this analysis since the sources that are not detected in Herschel images are likely the best candidates to reveal the quenching effect of powerful AGN winds on the star formation. Unfortunately, there are no public SED fitting codes that can properly deal with upper limit photometric points. Therefore we developed our SED fitting code, in which the best fit is the result of the sum of different components that describe the accretion disk emission, the unabsorbed stellar population, the emission of dust heated by the AGNs and by blue young stars. Each component is the result of the combination of many templates that we specify below. The weight $\mathrm{w}_{j}$ of each template can be found by minimizing the $\chi^{2}$ function

$\chi^{2}=\Sigma_{i}\left(\frac{\left(F_{i}-\Sigma_{j} w_{j} F_{i j}\right)}{\Delta F_{i}}\right)^{2}$,

where $F_{i}$ and $\Delta F_{i}$ are the fluxes and error measured in each photometric band and $F_{i j}$ is the flux in band $i$ for template $j$. Since this is a linear bounded problem, we determine the weight of each template using the idl routine BVLS.pro (bounded variable least-squares) by Michele Cappellari ${ }^{4}$. This routine solves the linear least-squares problem by finding the weights of each template in order to minimize the $\chi^{2}$ function. To estimate errors on fitting parameters, we adopt a Monte Carlo procedure. For each object we generate 1000 realizations of the observed SED with random extraction of photometric points normally distributed around the observed flux with sigma given by the errors. The photometric upper limits are treated as null detections with a positive error bar at $2 \sigma$ and we extract random values uniformly distributed in this range. For each realization of the observed SED we computed all the relevant physical quantities, for example the luminosity of each component $\left(L_{\mathrm{AGN}}, L_{\mathrm{SB}}\right.$, etc.). Finally, we estimate the errors as the percentile at $10 \%$ and $90 \%$ levels of the distribution of all the luminosities. If the best model does not require a starburst component, or the distribution is consistent with a null median value, we consider the 90 th percentile of the distribution as a $2 \sigma$ upper limit.

For the SED fitting for each component we adopt the following templates:

- the infrared emission of the starburst component was modelled with a linear combination of the Chary \& Elbaz (2001) templates. These models provide synthesized SEDs

4 http://www-astro.physics.ox.ac.uk/ mxc/software/ 

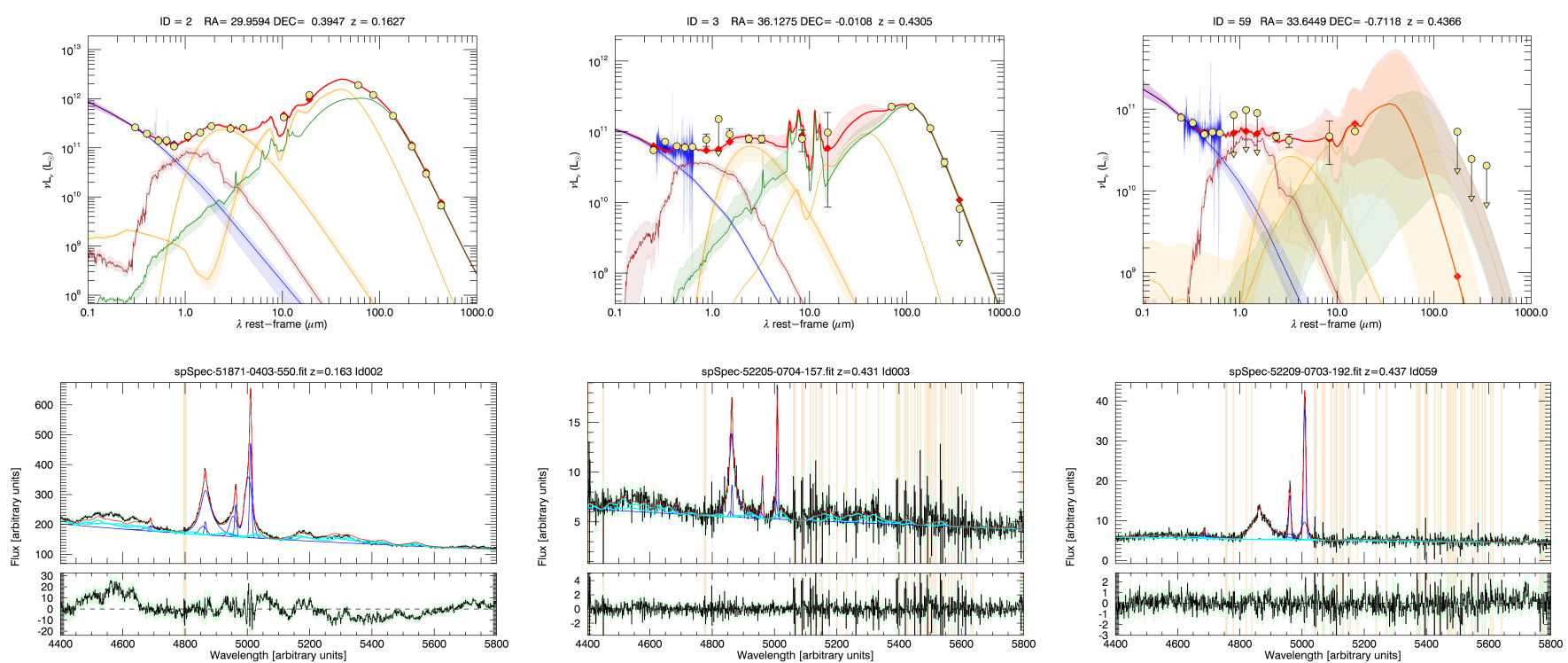

Fig. 4. Top panel: example of SED fitting for three quasars SDSS J015950.24+002340.8, SDSS J022430.60-000038.9, and SDSS J021434.78004242.6 of different SED and spectra quality. Points in yellow represent the luminosity of quasars (in solar luminosity units) from SDSS, 2MASS, WISE, PACS, and SPIRE images plotted versus the rest-frame wavelength (from 0.1 to $1000 \mu \mathrm{m}$ ). We plot in green the starburst template, in orange the torus and the hot black-body model, in brown the old star, and in blue the accretion disk component. The results of the composition is the best fit, shown in red. The hatched area represents the uncertainties for the normalization of each model components obtained from the Monte Carlo analysis. Bottom panel: example of the fit of the SDSS optical spectra for the same quasars presented above. In blue we plot the different components of the emission lines (narrow and broad) and in cyan the Fe emission lines template. In the bottom panel we show the residuals of the fit.

of 105 average SED templates of local luminous and ultraluminous purely star-forming infrared galaxies (LIRGs and ULIRGs) in a sequence of increasing infrared luminosity ${ }^{5}$;

- the AGN torus emission was modelled with a linear combination of DUSTY models by Nenkova et al. (2008). To select a few likely independent models (from more than $10^{5}$ ), we follow Roseboom et al. (2013) who demonstrate that it is possible to adequately fit their SED of luminous type 1 quasars from the SDSS survey with only three models. These three models are for tori with inclinations $i=0$ and $i=20$ (see Table 2 of their paper for details); to these we add the models at inclinations of $30,35,45,50,60,70$, and 75 degrees;

- the emission from three pure black bodies was modelled at temperatures 1100,1300 , and $1500 \mathrm{~K}$ in order to account for the hot dust emission in the near-infrared not adequately reproduced by torus models (e.g. Leipski et al. 2014);

- the stellar emission from an unobscured population was modelled with stellar templates from Bruzual \& Charlot (2003) computed with a Chabrier (2003) IMF and metallicities $Z=0.008,0.02,0.05$; we considered models with ages $5.1 \times 10^{8}, 2.0 \times 10^{9}, 5.0 \times 10^{9}, 1.0 \times 10^{10}, 1.3 \times 10^{10}$ years;

- the accretion disk emission was modelled with a linear combination of the four model spectra from Fig. 1 of Slone \& Netzer (2012) which are representative of a physical conditions of quasar;

- for the radio loud quasars it is possible that part of the infrared emission is dominated by synchrotron processes originating in the radio jets. However, the power-law slope of the sub-mm/far-infrared continuum is difficult to constrain in the

\footnotetext{
5 We have verified that the choice of the templates does not affect our results: adopting the starburst templates by Rieke et al. (2009) or Dale et al. (2014), the resultant SB and AGN luminosity differs by less than a factor of 3 .
}

infrared band. For this reason we prefer not to add a powerlaw component to the fitting templates and highlight the radio loud sources in all the plots, remembering that in some of these quasars the star formation rate may be overestimated.

In Fig. 4, top panel, we present examples of SED fitting.

We estimate the star formation rate from the starburst model in the rest frame $8-1000 \mu \mathrm{m}$ adopting a Chabrier initial mass function (Chabrier 2003). In detail we use the relation

$$
\left(\frac{S F R}{M_{\odot} \mathrm{yr}^{-1}}\right)=0.94 \times 3.88 \times 10^{-44}\left(\frac{L_{\mathrm{IR}}}{\operatorname{erg~s}^{-1}}\right)
$$

from Murphy et al. (2011). The factor 0.94 accounts for a slight SFR underestimation derived using the Chabrier (2003) IMF with respect to the Kroupa (2001) IMF (see Bolzonella et al. 2010; Pozzetti et al. 2010; Hainline et al. 2011). The stellar masses are derived from the normalization of the Bruzual \& Charlot (2003) templates, since these models are normalized to a total mass of one $M_{\odot}$ in stars. In Appendix A we test the accuracy of our SED fitting code, in particular with respect to the problem of model degeneracy.

\subsection{Fit of the optical spectra}

In this section we describe the analysis of the spectra used to reproduce the profile of the [O III] $\lambda 4959,5007$ and of the [O II] $\lambda 3727,3729$ emission line doublet in order to characterize the outflow properties. The reduced spectra data used in this work are available through the SDSS Data Archive Server 10 (DAS). The wavelength coverage is $\Delta \lambda \sim 3800-9200 \AA$ with a spectral resolution $R \sim 1800-2200(\Delta \lambda \sim 3600-10500 \AA$ and $R \sim 1400-2600$ for BOSS spectra).

We employed a $\chi^{2}$ minimization procedure using the IDL routine mpfit, Markwardt (2009). Since the optical emission 
lines show complex profiles diverging from a simple Gaussian profile, we fit the principal emission lines (i.e. the [O III] doublet, the narrow $\mathrm{H} \beta$ and eventually the He II $\lambda 4686$ with two Gaussians, one for a narrow component and one for the wings. The intensity ratio is fixed at [OIII] $\lambda 4959 /[\mathrm{O}$ III] $\lambda 5007=$ 0.33 (e.g. Dimitrijević et al. 2007) and the width of the [O III] $\lambda 4959$ line was fixed to be the same as the [O III] $\lambda 5007$ line and of the narrow $\mathrm{H} \beta$ line. The velocity offset of the narrow $\mathrm{H} \beta$ line are tied to those of the core [O III] $\lambda 4959,5007$ components. The [O II] emission line doublet in general is unresolved in our spectra. However, we fit the profile with two emission lines with the same $\sigma$ (each one made of two Gaussian components) with a flux ratio of the two components that vary between 1.3 and 1.5 .

The continuum is fitted with a power-law and the broad $\mathrm{H} \beta$ emission line with a broken power-law profile (Nagao et al. 2006). A major difficulty in the spectral analysis is that the [OIII] doublet overlaps with the broad $\mathrm{H} \beta$ emission and optical Fe II emission lines (see Fig. 4, bottom panel). The Fe II emission was modelled with a linear combination of templates, which was then convolved with a broadening Gaussian function. The free parameters characterizing the Fe II emission are the weights of the template, the central velocity, and the velocity dispersion of the Gaussian function. The adopted Fe II templates were the observed IZw1 spectrum (Véron-Cetty et al. 2004).

In many cases the $[\mathrm{O} \mathrm{III}]$ and $[\mathrm{O} \mathrm{II}]$ lines are too weak and confused with the continuum and the other fitting components to extract reliable kinematic information. Therefore, we adopt thresholds in flux and in $\mathrm{S} / \mathrm{N}$ to discard useless spectra. We only consider spectra with

$-S / N>5$ for the emission line flux;

$-\log \left(F_{[\mathrm{O} \text { III }]}\right)>-11.5 \mathrm{erg} \mathrm{s}^{-1} \mathrm{~cm}^{-2}$.

For the $[\mathrm{OII}]$ line we lower the flux threshold to $-12.5 \mathrm{erg} \mathrm{cm}^{-2} \mathrm{~s}^{-1}$, because this line is weaker and not blended with other strong features. We exclude an additional 15 sources with high $\mathrm{S} / \mathrm{N}$ and high $F_{[\mathrm{O} \text { III] }}$, but with the [O III] line blended with Fe lines or $\mathrm{H} \beta$ and with a flux that is negligible with respect to the other components. The presence of optical Fe emission lines in SDSS quasar spectra has been systematically investigated by $\mathrm{Hu}$ et al. (2008). They found that Fe II emission lines are typically redshifted with respect to the [O III] peak, and they suggest that the optical Fe emission lines trace gas in the broad line region dominated by infall. In Fig. 5 we represent our selection criteria on [O III] plotting the flux versus the $\mathrm{S} / \mathrm{N}$ of the line. Red points represent the rejected quasars and green points quasars with bona fide clear lines and reliable fits to the profile (confirming by eye the quality of our selection criteria). Adopting this approach, we define a high quality subsample 1, composed of 125 QSOs with trustworthy fitted profiles in [O III] (out of 224 QSOs), and a high quality subsample 2, made of 108 QSOs with good quality fits both in $[\mathrm{O} \mathrm{III}]$ and $[\mathrm{O} \mathrm{II}]$. These quasars are used in the plots involving [O II] in addition to [O III] (we have respectively $19 \%$ and $18 \%$ of radio loud quasars in the two subsamples). In the following analysis we extract the outflow properties (on a case-by-case basis using the [O III], the [O II], or sometimes both the line profiles) considering only the quasars that satisfy our quality selection criterium. In Fig. 6 we plot the histogram of the star formation rate in the complete sample and in our high quality spectra sample, highlighting the objects with star formation rate upper limits. The fraction and the distribution of SFR undetected objects is of great interest, since in these objects the SF-quenching might be ongoing. In the complete sample $38 \%$ of

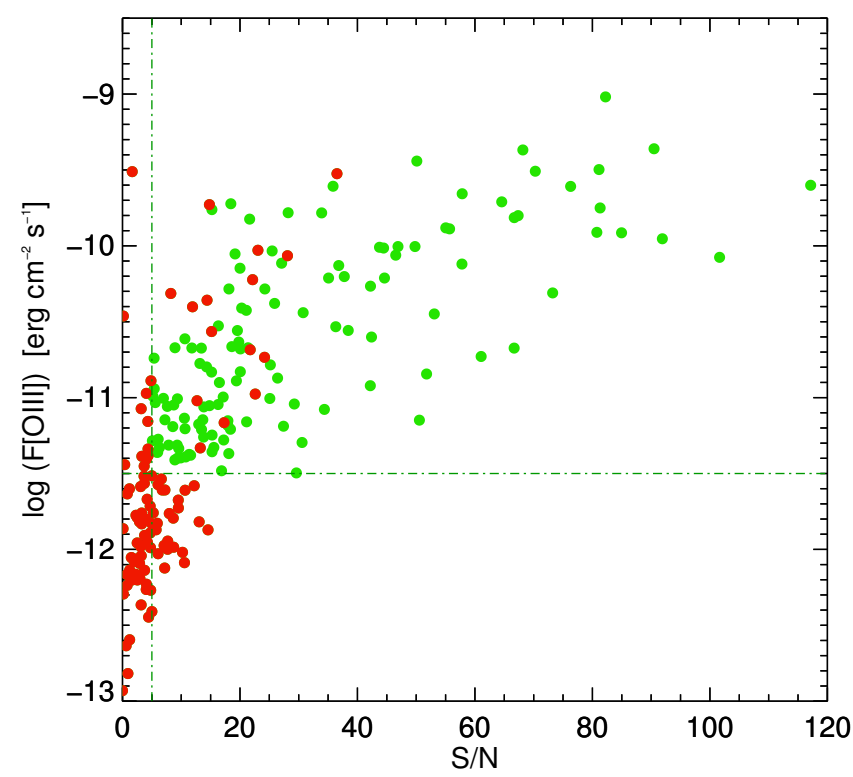

Fig. 5. Logarithmic flux (in $\mathrm{erg} \mathrm{s}^{-1} \mathrm{~cm}^{-2}$ ) versus the signal-to-noise ratio of the [O III] emission line. We adopt the dotted line as the threshold in flux and $\mathrm{S} / \mathrm{N}$ to select only spectra with a visible [O III] emission line and reliable fit (green points). Red points represent the quasars for which we consider the kinematic parameters based on unreliable [O III] lines and we do not consider these QSO in the following analysis.

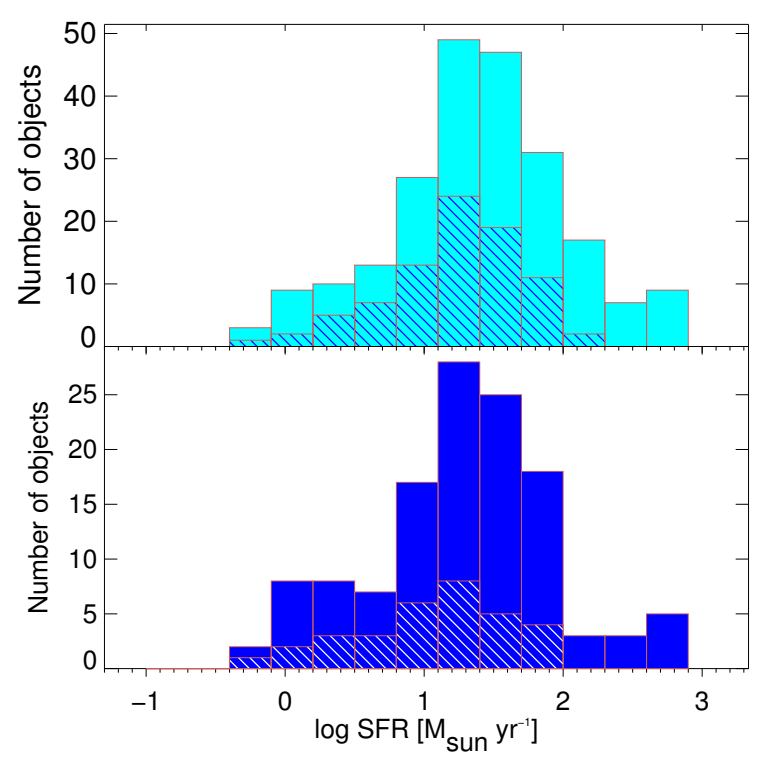

Fig. 6. Histogram of the star formation rate distribution for all the quasars (top panel) and only for quasars with reliable [O III] measurements. The hatched area represent quasars with upper limit values on SFRs.

the quasars have a star formation rate upper limit measurement. This fraction decreases to $26 \%$ in the subsample of quasars with reliable [O IIII] measurements. We note that although the sensibility of our observations is almost heterogeneous, the distribution of the IR luminosity for detected and undetected quasars is similar and the IR luminosity upper limits are not located in the tail of the distribution of detected quasars.

Owing to the uncertainties in the decomposition of the line into a narrow and a broad component, we follow the approach of other authors (e.g. Harrison et al. 2014; Perna et al. 2015) and we 

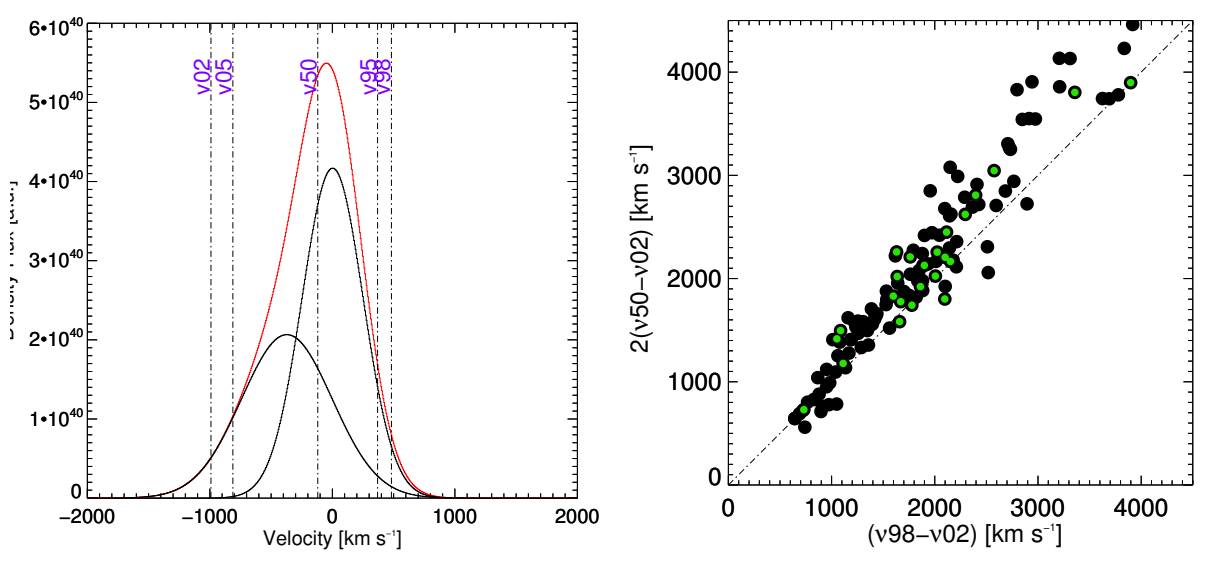

Fig. 7. Left panel: example of [OIII] $\lambda 5007$ line decomposition in a narrow and broad component and of different velocity definition used to characterize the properties of the outflow. In particular we show velocities at different percentiles of the flux contained in the overall emission line profile (from left to right: 2nd; 5th, 50th, 95th, and 98th). Right panel: two times the blue tail, defined as (v50-v02) versus maximum velocity of the [O III] line $(v 98-v 02)$. The dashed line is the bisectrix of the plane and represents the condition for symmetry. Almost all of the objects show a broad blue tail. Green points are for radio loud quasars.

adopt a non-parametric definition for the velocity characterizing the outflow. We reconstructed a synthetic line profile adding two Gaussian lines and we define different percentiles of the flux contained in the overall emission profile. In particular we measure the velocity at the $2 \mathrm{nd}, 5 \mathrm{th}, 50 \mathrm{th}, 95 \mathrm{th}$, and 98 th percentile, containing respectively $2,5,50,95$, and $98 \%$ of the overall line flux $(v 02, v 05, v 50$, etc.). The standard deviation $(\sigma)$ of the line profile is the square root of the second moment about the mean. In Fig. 7, left panel, we illustrate these definitions using an example of a [O III] $\lambda 5007$ line profile.

In the following analysis, we select the following three parameters to describe the outflow properties and to explore the connection with the SFR:

- $v_{\text {blue }}$, the maximum velocity of the blue wing, defined as $\left|v_{10}-v_{50}\right|$, linked in some complex way to the energetic of the outflow. In principle, one would infer the mass outflow rate, but this measurement is highly uncertain and requires the knowledge of the location, mass density, geometry, and velocity of the gas outflowing. Assuming simple geometric arguments, the mass outflow rate is proportional to the maximum velocity of the outflowing wind $(\sim 3 \mathrm{vM} / \mathrm{R}$, e.g. Maiolino et al. 2012).

- $\Delta v_{\text {offset }}$, the velocity offset of the broad wings defined as $\Delta v_{\text {offset }}=\left|v_{05}+v_{95}\right| / 2-v_{50}$. For two well-separated Gaussian components, $\Delta v_{\text {offset }}$ is the velocity offset of the broad component with respect to $v_{50}$ (Harrison et al. 2014). As usual, in the literature we interpret the broad, blueshifted emission line with respect to the velocity of the [O III] narrow component as the result of an outflowing gas moving at larger velocity with respect to the quieter large-scale gas.

$-\Delta v_{\sigma[\mathrm{O} \text { III }]}=\left(\sigma_{[\mathrm{O} \text { III }]}^{2}-\sigma_{[\mathrm{O} \text { II }]}^{2}\right)^{0.5}$, the sigma excess, defined as the square root of the quadratic difference between the standard deviation of the [O III] and [O II] lines. This parameter estimates the different level of the perturbation of the gas in the inner and outer portion of the NLR since, as we discuss in Sect. 4.1, the bulk of the [O III] emission should be produced in a more inner region than [O II] .

\section{Results}

\subsection{Comparison of the [O III] and [O II] line profile}

In the literature a blueshifted line seen in absorption is considered a clear signature of outflow. Instead, the interpretation of blueshifted wings seen in emission is ambiguous because different scenarios can account for the asymmetry of the line: outflows, inflows, or gas in a rotating disk partially obscured by an asymmetric distribution of dust. Moreover, if the extinction is within the emitting clouds, then outflowing gas would produce a line profile with a red wing (Whittle 1985; De Robertis \& Shaw 1990; Veilleux 1991). However, in recent papers a blueshifted wing is considered a clear signature of ionized gas moving towards our line of sight (e.g. Bian et al. 2005; Komossa et al. 2008; Brusa et al. 2015; Villar-Martín et al. 2011a). Here we follow the latter interpretation, aware that all our results depends on this assumption.

In Fig. 7, right panel, we emphasize the asymmetry of the [O III] emission lines plotting $v_{\max }=v_{98}-v_{02}$ versus two times the blue tail (defined as $v_{50}-v_{02}$ ). The deviation from the bisectrix of the plane represents the amount of asymmetry with respect to $v_{50}$. About $80 \%$ of the quasars show asymmetric [O III] emission lines with significant blue wings. The median deviation from symmetry is $211 \mathrm{~km} \mathrm{~s}^{-1}$ but it increases up to $1033 \mathrm{~km} \mathrm{~s}^{-1}$ at higher $v_{\max }$.

In various models of line formation in the NLR, the larger widths of the ionized lines is explained by their formation in clouds that have higher density or higher orbital or radial velocity, likely because they are closest to the central ionization region (De Robertis \& Osterbrock 1984) ${ }^{6}$. The larger width of the $[\mathrm{O} I I I]$ with respect to $[\mathrm{O} I \mathrm{II}]$ is consistent with the fact that lines of higher ionization potential and/or higher critical densities are produced in inner regions closer to the nucleus. If the [O II] emitting gas likely traces the unperturbed ISM, the excess of the standard deviation of the [O III] line with respect to [O II] is an estimate of the amount of perturbation of the gas in inner regions.

In Fig. 8, left panel, we plot the sigma values derived from the analysis of the [O III] and [O II] emission line profile. The $\sigma_{[\mathrm{O} \text { III }]}$ values span a range in velocity between 165 and $950 \mathrm{~km} \mathrm{~s}^{-1}$, with a mean value of $405 \pm 165 \mathrm{~km} \mathrm{~s}^{-1}$. For comparison, Mullaney et al. (2013) in a sample of type 1 quasars found a value of the order of $195 \mathrm{~km} \mathrm{~s}^{-1}$ (we derived this value combining their average two-Gaussian component with FWHM of 335 and $851 \mathrm{~km} \mathrm{~s}^{-1}$ ). The value of $\sigma_{[\mathrm{O} \mathrm{III]}}$, except for a few cases, is always larger than $\sigma_{[\mathrm{O} I]}$. For the [O II] emission line we find $\sigma_{[\mathrm{O} \text { II] }}=185 \pm 70 \mathrm{~km} \mathrm{~s}^{-1}$, a value comparable with the stellar velocity dispersion, which in quasars is of the order of 200-300 $\mathrm{km} \mathrm{s}^{-1}$ (depending on the black hole masses).

In order to characterize the gas outflow velocity with respect to the unperturbed gas at galactic scales it is essential to determine an accurate velocity reference. In our quasar sample the

6 The critical density and the ionization potential for [OIII] is $7 \times 10^{5} \mathrm{~cm}^{-3}$ and $\chi_{e v}=35.1$, higher than the critical density for the [OII] doublet $\left(1.4-3.3 \times 10^{3} \mathrm{~cm}^{-3} \mathrm{eV}\right)$ and the ionization potential for $[\mathrm{O} I \mathrm{II}]\left(\chi_{e v}=13.6 \mathrm{eV}\right)$, Nagao et al. $(2001)$. 

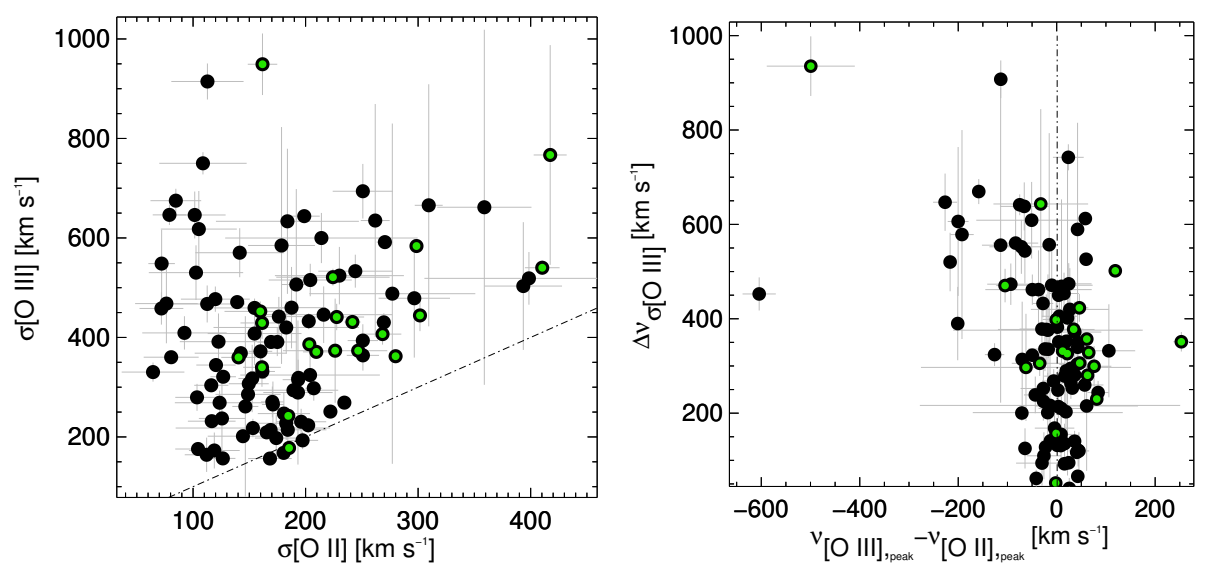

Fig. 8. Comparison of the [OIII] and [OII] principal emission line parameters. Left panel: sigma of the [O III] line versus the sigma of the [O II]. The [OIII] lines show broader widths. Right panel: $\sigma$ excess (defined using the square root of the quadratic difference of the $[\mathrm{O} \mathrm{III]}$ and [O II] sigma, see text) versus the relative shift of the [O III] peak respect to [O II] peak (both referring to their rest frame position). stellar absorption-line features cannot be detected, because the continuum is dominated by the AGN. The velocity of the core of the [O III] line has been used to estimate the systemic redshift of the galaxy (Villar-Martín et al. 2011b) and the width of the line has been considered a reasonable substitute for the stellar velocity dispersion of the galaxy bulge (after properly removing the asymmetric blue wings of the line, Greene \& Ho 2005). Assuming that the outer portion of the NLR gas follows pure gravitational motions, we test whether the velocity of the [O II] line can be used as a reference velocity for large scale gas.

In Fig. 8, right panel, on the $x$-axis we show the shift in velocity of the [O III] peak flux density with respect to the [O II] peak both referring to the rest frame wavelength. We measure a mean shift of the two peaks of $\left\langle V_{\text {shift }}\right\rangle=-20 \pm$ $100 \mathrm{~km} \mathrm{~s}^{-1}$. Unfortunately, the [OII] doublet is unresolved in the SDSS spectra. The ratio of the two lines depends on the electron density of the gas and it is between 0.35 and 1.5 (Pradhan et al. 2006). This introduces an uncertainty in our [OII] velocity reference of $2 \AA$, corresponding to $\sim 80 \mathrm{~km} \mathrm{~s}^{-1}$. For most of the quasars we find relative shifts of the two peaks in this range. However, a few quasars ( $20 \%$ of the total) show velocity shifts larger than $80 \mathrm{~km} \mathrm{~s}^{-1}$. For comparison, on the $y$-axis we show $\left.\Delta v_{\sigma[\mathrm{O}} \mathrm{III}\right]$. The quasars that show larger [O III] shift with respect to [O II] also have larger $\Delta v_{\sigma[\mathrm{O} \text { III }]}$ values. We believe that this approach is promising; unfortunately, the [O II] doublet is unresolved in our spectra and so we prefer not to use the peak of this line as a reference frame and we choose other parameters to characterize the outflow properties.

\subsection{SB and AGN dominated quasars}

The total infrared luminosity between $8-1000 \mu \mathrm{m}$ is the sum of different contributions: the torus and dust heated by newly formed stars. We divide our sample into two groups according to the dominant processes that produce the infrared-luminosity. The first group is composed of starburst dominated objects for which the starburst-related luminosity dominates the total infrared $(8-1000 \mu \mathrm{m})$ luminosity $\left(L_{\mathrm{SB}} / L_{\mathrm{tot}}>0.5\right)$ and the second group is composed of AGN component dominated objects $\left(L_{\mathrm{SB}} / L_{\mathrm{tot}}<0.5\right)$ (or quasars with upper limits in SFR). In this section we explore the properties of these two groups (hereafter SFd-QSO and AGNd-QSO).

We measured the star formation rate from SED fitting in the infrared band; however, the [O II] emission line, corrected for dust extinction, is another widely used estimator of the SFR. Deriving the SFR from the [O II] luminosity is quite a challenging task because in type 1 quasars the [O II] luminosity is
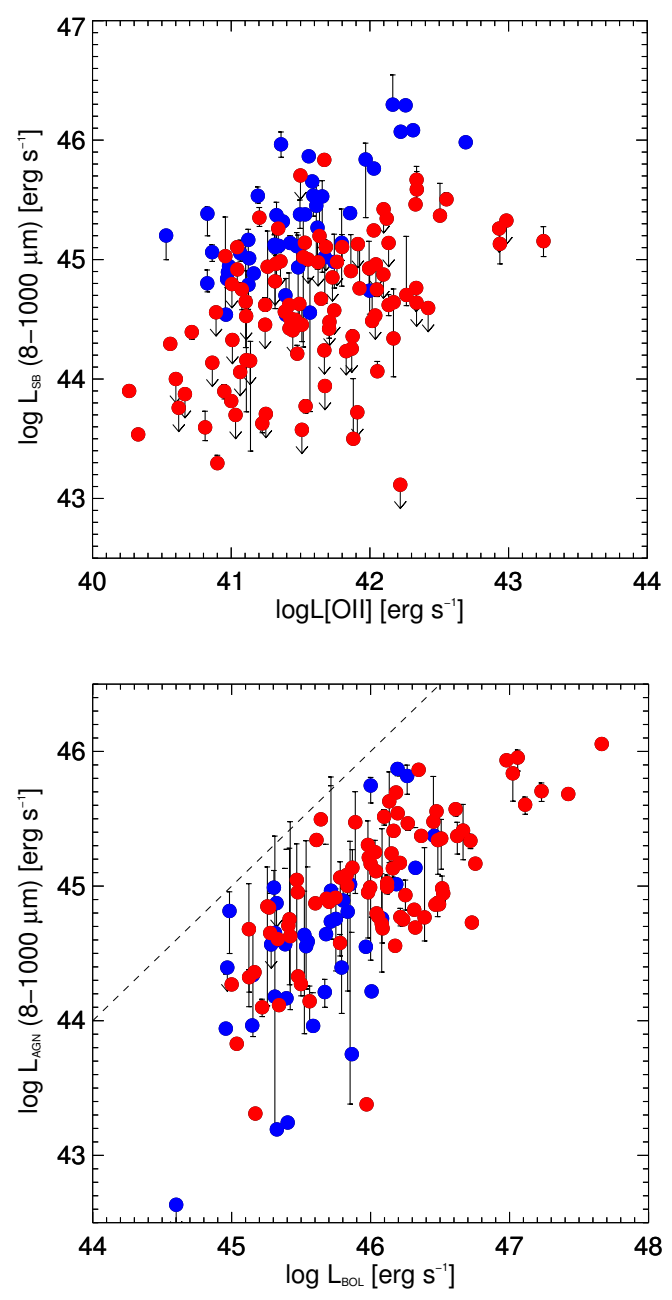

Fig. 9. Upper panel: logarithm of the infrared luminosity in the 8-1000 $\mu \mathrm{m}$ range for the starburst component derived from the SED fitting versus the logarithm of the [OII] luminosity, another estimator for the SFR. Bottom panel: logarithm of the infrared luminosity in the $8-1000 \mu \mathrm{m}$ range for the hot dust component derived from the SED model (likely produced by a dusty torus warm up by the AGN) versus the logarithm of the AGN bolometric luminosity. Blue and red points represent quasars dominated in the infrared by star formation or by the AGN emission, respectively.

contaminated by the emission produced in the NLR. Moreover, the conversion from luminosity to SFR has a complex dependence on metallicity (Kewley et al. 2004). In Fig. 9, upper panel, we compare the [O II] versus the infrared luminosity in 


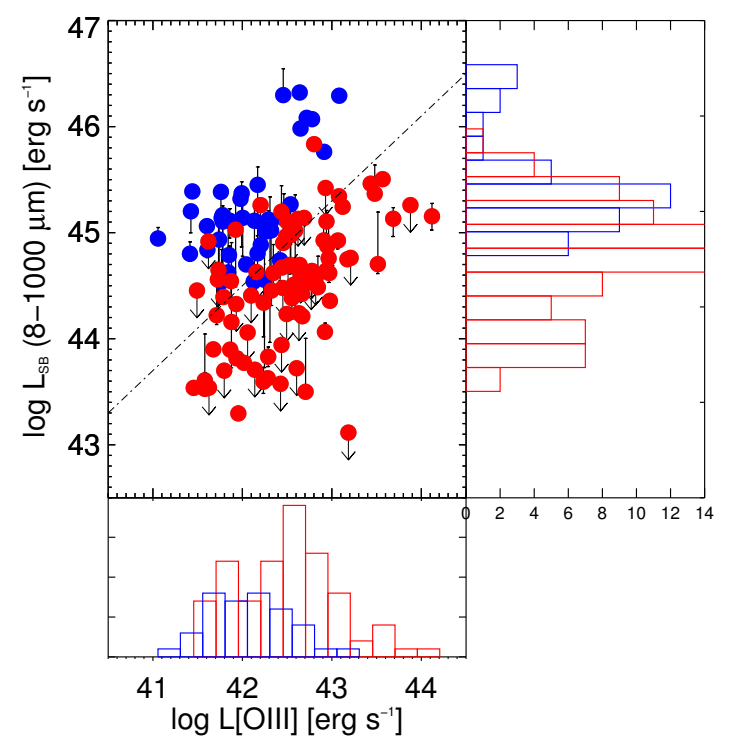

Fig. 10. Logarithm of the star formation rate versus the logarithm of the [OIII] luminosity. The dashed line shows the correlation by Netzer (2009) of slope 0.8. The normalization is from Shao et al. (2010) dividing the AGN bolometric factor by a factor of 3500. The histograms at the right and bottom represent the distribution of [OIII] luminosity and of star formation rates.

the $8-1000 \mu \mathrm{m}$ derived from SED fitting. The [O II] luminosity is not corrected for dust absorption because only about half of our spectra $(z \lesssim 0.4)$ cover the Balmer decrement $(\mathrm{H} \alpha / \mathrm{H} \beta)$ typically used to make the correction. As expected, the dispersion is larger if we consider all the quasars, but it is significantly reduced if we consider only the SFd-quasars. The red points (AGNd-quasars, 102 objects) may have $L_{[\mathrm{O} \text { II] }}$ significantly contaminated by the AGN, and in fact they show an excess of [O II] luminosity with respect to the blue points (SFd-dominated quasars, 46 objects).

In the bottom panel of Fig. 9 we compare the $8-1000 \mu \mathrm{m}$ luminosity for the AGN component versus the AGN bolometric luminosity (derived from $L_{[\mathrm{O} I I I]}$ as $L_{\mathrm{BOL}}=3500 \times L_{[\mathrm{O} I I]}$, Heckman et al. 2004). The ratio between these two quantities provides an estimate of the torus covering factor. We find a mean logarithmic value of $<-1 \pm 0.5$, consistent with the estimate of $-0.4 \pm 0.2$ of Roseboom et al. (2013) measured in a sample of type 1 quasars (our [O III] luminosity measurements are not corrected for dust absorption and therefore we consider them as lower limit values). In the AGNd-quasars sample (86 objects), the $L_{[\mathrm{O} \text { III }]}$ correlates with the infrared emission derived from torus models (we find a linear correlation coefficient of $r=0.6)$. Instead we do not find a relation in the SFd-quasars sample (39 objects, $r=0.2)$.

In Fig. 10 we investigate the relation between the AGN [O III] luminosity and the star formation rate of the host galaxy of the two groups. There is a well-known close correlation between the AGN bolometric luminosity and the star formation rate of galaxies (e.g. Netzer 2009). This relation is usually interpreted as a probe of the coupling between AGN growth and the star formation by an evolutionary mechanism, e.g. the merging. Our quasars recover the correlation found by Netzer (2009) $L_{\mathrm{AGN}} \propto L_{S F R}^{0.8}$ that links the AGN luminosity to the SFR.

\subsection{SB and outflow properties}

We now consider possible relations between the SFR and the kinematic parameter characterizing the outflowing gas and we discuss these results in a general context in Sect. 6. We focus first on the SFd-QSO sample (Fig. 11, top panel). We find an upward trend between the logarithm of the SFR and the three kinematic parameters considered, but no significant correlation. For the AGNd-QSO, we do not find a trend between the outflow properties and the SFR. In Fig. 11, bottom panel, we consider the SFR versus the three same kinematic parameters considered in the top panel. Even separating the radio loud and radio quiet quasars we did not find a relation between the SFR and kinematics of the ionized gas, as expected if the dominant source powering the outflow are supernovae winds or the AGN. We then searched for a connection between the kinematics of the ionized gas and the properties of the AGN (e.g. $L_{[\mathrm{O} \mathrm{III}]}, L_{\mathrm{tot}(8-1000 \mu \mathrm{m})}$, $L_{\text {Edd }}$ ), but again we do not find any linear relations.

\subsection{Testing the negative AGN feedback model}

The SFR of star-forming galaxies correlates with the stellar mass in the local Universe, as well as in the distant Universe out to $z \sim 2$ (e.g. Noeske et al. 2007; Daddi et al. 2007; Elbaz et al. 2007). This close correlation (dispersion of $\sim 0.3 \mathrm{dex}$ ) is called the main sequence and it can be used to define the outliers, i.e. quenched and starburst galaxies. Later on, the specific star formation rate (sSFR), defined as the SFR per unit stellar mass, is used to investigate the dependence of the SFR with many parameters characterizing the galaxies or the environment. For example, a dependence of the sSFR on stellar mass and redshift (Bauer et al. 2005) was discovered. Galaxies at redshift equal to one tend to have star formation rates that are a factor of ten higher than the local ones. Also, high redshift galaxies are typically more massive than low-redshift star-forming galaxies (Seymour et al. 2008).

In this work, we compute the SFR in redshift bins independently for strong and weak outflow galaxies (assuming similar stellar masses in each redshift bin for the two groups). In order to divide the quasars into the two groups, we consider the distribution of $v_{\text {blue }}, \Delta v_{\text {offset }}$ and $\Delta v_{\sigma[\mathrm{O} \text { III }]}$ and as the threshold we choose the median of the respective distributions $\left(v_{\text {blue }}>556\right.$, $\Delta v_{\text {offset }}>166$ and $\Delta v_{\sigma[\mathrm{O} \text { III }]}>335 \mathrm{~km} \mathrm{~s}^{-1}$ ), see Fig. 12. In Fig. 13 we show the logarithm of the SFR versus redshift plotting strong and weak outflow quasars according to these three criteria. We have separated the quasars into four redshift bins $(z<0.2,0.2<z<0.4,0.4<z<0.6$, and $z>0.6$, see Table 1$)$ and in each redshift bin we compare the mean star formation rate for the two classes separately, taking error bars and upper limits in the SFR measurements into account.

It is not an easy task to calculate a weighted mean with upper limits in a dataset. We adopt the following strategy. For each quasar we consider the histogram distribution of SB luminosity (i.e. the distribution of the luminosity measurements obtained through a Monte Carlo analysis). Some of the QSO are considered upper limits and their distribution peaks at zero luminosity, others are detections and their distribution resemble a Gaussian distribution. We randomly extract $10^{4} \mathrm{SB}$ luminosities from the distributions of all quasars separating them in four classes of redshift and in strong and weak outflow quasars. In these four redshift bins, we obtain two histograms of the total luminosities that take into account detected and undetected sources. We evaluate the mean and the error at the $10 \%$ and $90 \%$ percentiles of the distribution and we overplot the median points in the figure. Since we may have overestimated the star formation rate for radio loud quasars, and the type of the AGN feedback on the host could be different, we repeat the same analysis excluding radio loud quasars (Fig. 13, bottom panel). 

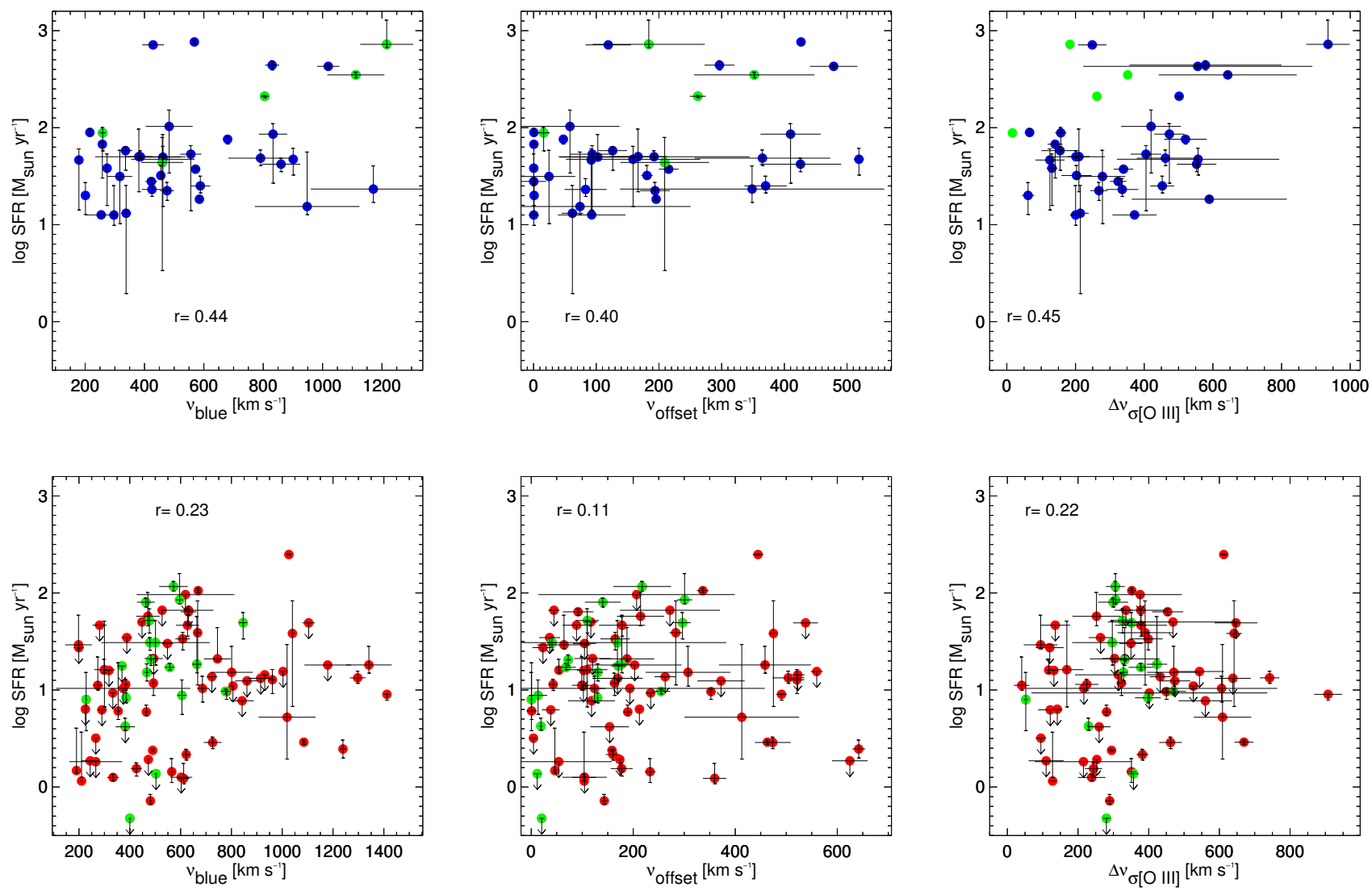

Fig. 11. Relation between the SFR and the three parameters characterizing the outflow ( $\left.v_{\text {blue }}, \Delta v_{\text {offset }}, \Delta v_{\sigma[0 \text { IIII }]}\right)$ for the starburst dominated quasars (blue points, top panel) and the AGN dominated quasars (red points, bottom panel). There is no clear relation between outflow and SF considering the AGN and SB dominated quasars. If we consider only the SFd-dominated objects (blue points) a positive trend emerges, although with a weak significance and a large scatter.
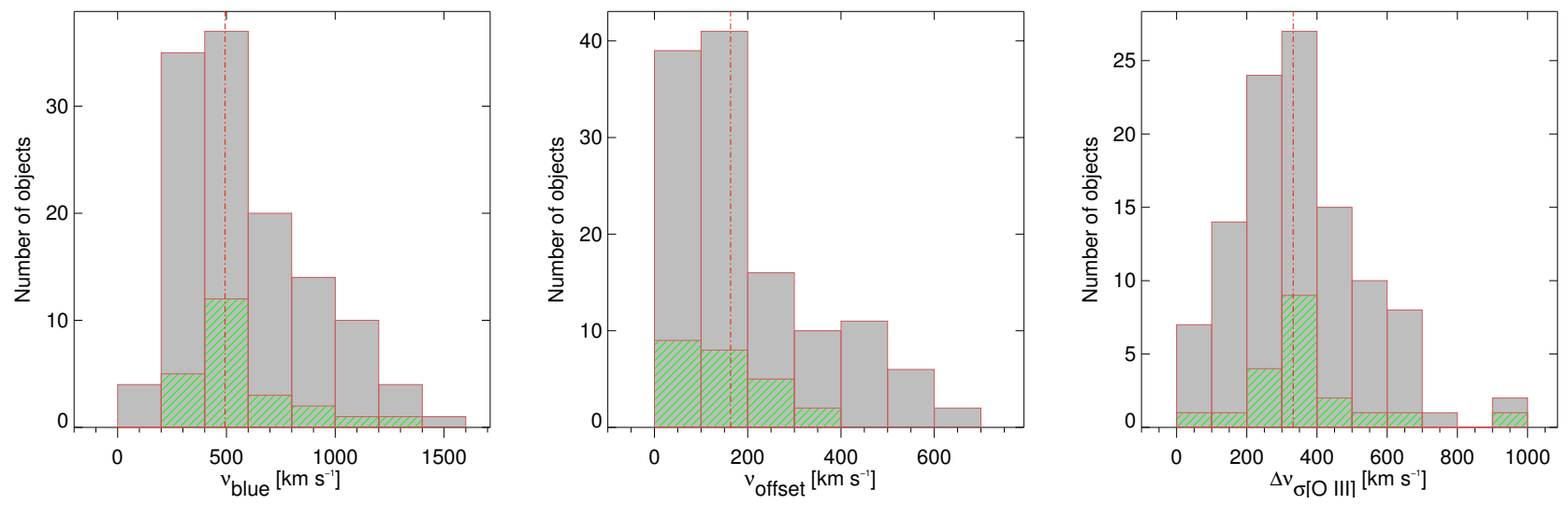

Fig. 12. Histogram of the distribution of the three parameters characterizing the outflow. The red dashed line marks the median of the distribution. From left to right: $v_{\text {blue }}, \Delta v_{\text {offset }}, \Delta v_{\sigma[\mathrm{O} \text { III] }}$. The green hatched area represents the radio loud quasars.

We note an increase in the mean star formation rate with redshift with a similar slope in the two groups. This is expected because it is well known that the cosmic star formation rate peaks around $z \sim 2-3$, after which it decreases by an order of magnitude to the present Universe (e.g. Hopkins et al. 2006). More importantly, we find that the star formation rate is similar in the two groups of quasars and, typically, strong-outflow quasars show values only slightly larger than weak-outflow quasars, even excluding the radio loud quasars. In each redshift bin the discrepancies between the median values in the two groups are slightly reduced if radio loud galaxies are excluded from the analysis. We have found no evidence that the $\mathrm{SF}$ in the host is suppressed in the presence of strong outflows comparing the SFR in bin of redshift in quasars characterized by strong or weak outflow signatures.

\section{Main sequence of quasar host galaxies}

One of the best ways to take into account the effects due to different galaxy masses is to normalize the SFR to the stellar mass obtaining the so-called specific star formation rate (sSFR). However, given the difficulties in measuring stellar masses in 
B. Balmaverde et al.: Ionized gas outflow and star formation
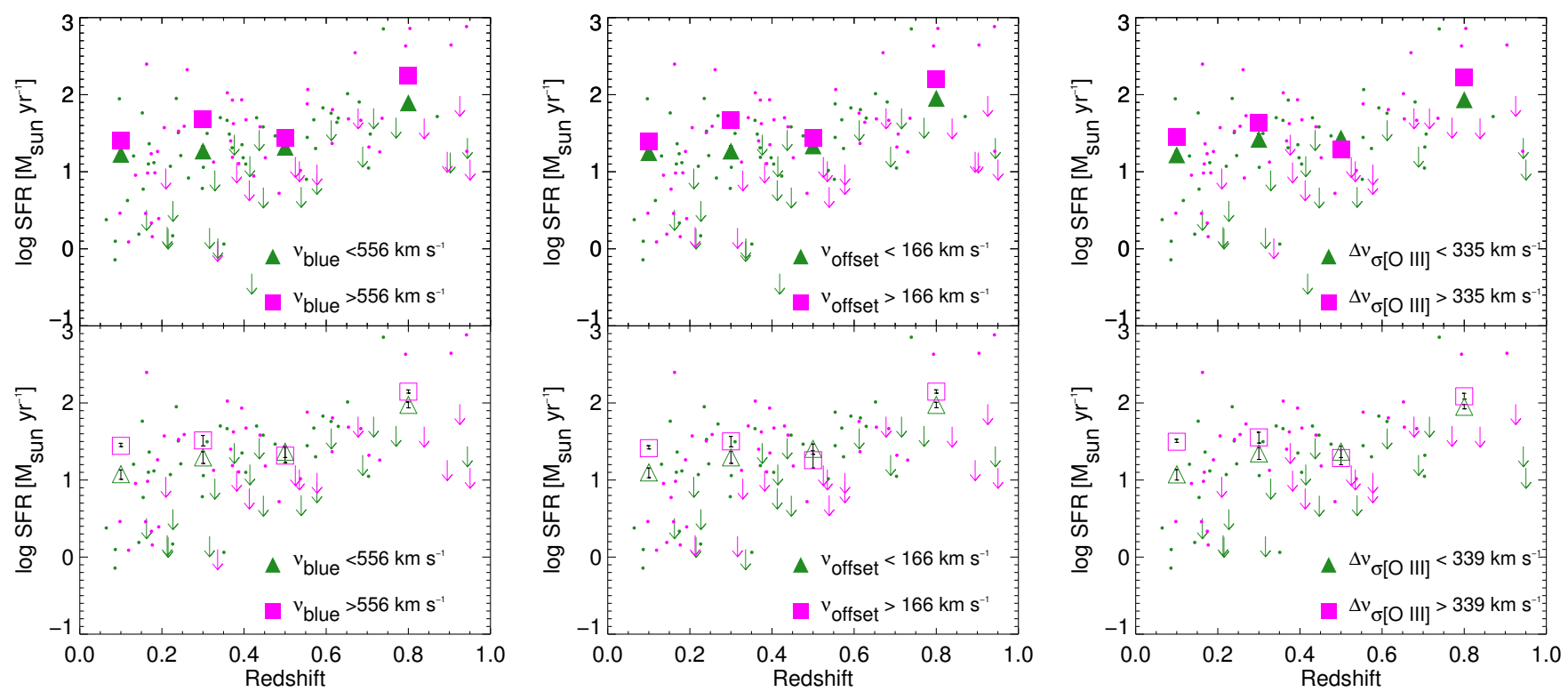

Fig. 13. Upper panel: star formation rate plotted in four bins of redshift for the complete sample of quasars. We adopt different criteria for separating strong-outflow (small magenta points) and weak-outflow galaxies (small green points), respectively higher and lower than the median value of the distribution of the three parameters ( $v_{\text {blue }}$ (first panel), $\Delta v_{\text {offset }}$ (second panel), and $\left.\Delta v_{\sigma[0 \text { III }]}\right)$. Bottom panel: same as top, but excluding radio loud quasars.

Table 1. Statistic results for the SFR plotted in bin of redshift (see Fig. 13).

\begin{tabular}{l|llll}
\hline \hline \multicolumn{2}{|c}{ Weak-out. QSO } & \multicolumn{2}{c}{ Strong-out. QSO } \\
$z$ bin & Det/ul & $\langle S F R\rangle$ & Det/ul & $\langle S F R\rangle$ \\
\hline$v_{\text {blue }}$ & \multicolumn{5}{c}{} \\
\hline $0-0.2$ & $12 / 1$ & $1.23(1.07)$ & $12 / 0$ & $1.41(1.44)$ \\
$0.2-0.4$ & $16 / 7$ & $1.27(1.29)$ & $13 / 3$ & $1.69(1.51)$ \\
$0.4-0.6$ & $9 / 6$ & $1.32(1.37)$ & $8 / 4$ & $1.44(1.31)$ \\
$0.6-1$ & $9 / 7$ & $1.89(2.01)$ & $13 / 5$ & $2.25(2.15)$ \\
\hline$\Delta v_{\text {offset }}$ & & & & \\
\hline $0-0.2$ & $12 / 1$ & $1.25(1.10)$ & $12 / 0$ & $1.39(1.41)$ \\
$0.2-0.4$ & $16 / 6$ & $1.27(1.29)$ & $13 / 4$ & $1.67(1.50)$ \\
$0.4-0.6$ & $11 / 5$ & $1.34(1.40)$ & $6 / 5$ & $1.43(1.26)$ \\
$0.6-1$ & $7 / 6$ & $1.95(1.97)$ & $15 / 6$ & $2.20(2.15)$ \\
\hline$\Delta v_{\sigma[0}$ III] & \multicolumn{5}{|c}{} \\
\hline $0-0.2$ & $10 / 1$ & $1.22(1.07)$ & $12 / 0$ & $1.45(1.50)$ \\
$0.2-0.4$ & $13 / 5$ & $1.42(1.34)$ & $11 / 4$ & $1.63(1.55)$ \\
$0.4-0.6$ & $9 / 5$ & $1.44(1.36)$ & $6 / 5$ & $1.29(1.28)$ \\
$0.6-1$ & $7 / 5$ & $1.93(1.95)$ & $10 / 5$ & $2.18(2.09)$ \\
\hline
\end{tabular}

Notes. For the three kinematic parameters adopted, we report in each bin of redshift (Col. 1) the number of the detection and upper limits and the mean SFR in logarithmic scale (in parenthesis the value obtained excluding radio loud quasars) for unperturbed quasars (Cols. 2 and 3) and outflow dominated quasars (Cols. 4 and 5).

quasar host galaxies from SED fitting in the IR band caused by the strong AGN contamination, we adopt this approach as a consistency check. The offset from the SF main sequence provides evidence that the SF in the galaxy is enhanced or quenched with respect to most of the galaxies. Therefore, we derive the stellar mass from the normalization of the Bruzal \& Charlot stellar template.
Table 2. Results of the correlation analysis.

\begin{tabular}{llll}
\hline \hline Variables & Disp. & $\mathrm{r}(N)$ & Sign. \\
\hline Agn dominated quasars & & & \\
\hline log SFR vs. Max velocity & 0.2 & $0.44(39)$ & $95 \%$ \\
SFR vs. Wings vel. offset & 0.2 & $0.40(39)$ & $95 \%$ \\
log SFR vs. $(\sigma$ [OIII] $-\sigma[\mathrm{OII}])$ & 0.2 & $0.45(33)$ & $95 \%$ \\
\hline SB dominated quasars & & & \\
\hline log SFR vs. Max velocity & 0.5 & $0.23(86)$ & NO \\
log SFR vs. Wings offset & 0.5 & $0.11(86)$ & NO \\
log SFR vs. $\Delta$ VSIG [OIII] & 0.5 & $0.22(75)$ & NO \\
\hline
\end{tabular}

Notes. Column 2: the variance of the intrinsic scatter. Column 3: the linear correlation coefficient between the dependent and independent variables (Pearson product-moment correlation coefficient). The maximum positive (negative) correlation is indicated by a correlation coefficient of $1(-1)$. An absolute value between 0.4 and 0.7 could indicate moderate association. Column 4: significance of the linear correlation. A value of $P$ less than $0.01(0.05)$ imply a significant measurement at $1 \%(5 \%)$, id.e. there is $1 \%(5 \%)$ percentage chance that the finding is non-significant. $N$ is the size of the sample.

As mentioned above, measuring stellar masses in type 1 AGNs and in quasars in particular is extremely challenging. However, in many cases the near IR SEDs of our sources around $1-3 \mu \mathrm{m}$ are dominated by the emission of old stars, allowing a reliable estimate of the stellar mass. We consider convincing the stellar mass derived from SED fitting in which the luminosity of the old star components contribute at least $25 \%$ to the total luminosity in the 1-3 $\mu$ m range. We show in Fig. 14, right panel, the distribution of the stellar mass values obtained for the whole sample of quasars (the dashed area represents uncertain measurements, about $30 \%$ of the total measurements). By considering only the reliable values we obtain a mean value of $10^{10.8} M_{\odot}$, consistent with the range found by Mainieri et al. (2011) between $8 \times 10^{9}$ and $10^{11} M_{\odot}$ for a sample of type 2 quasars from the COSMOS survey. 

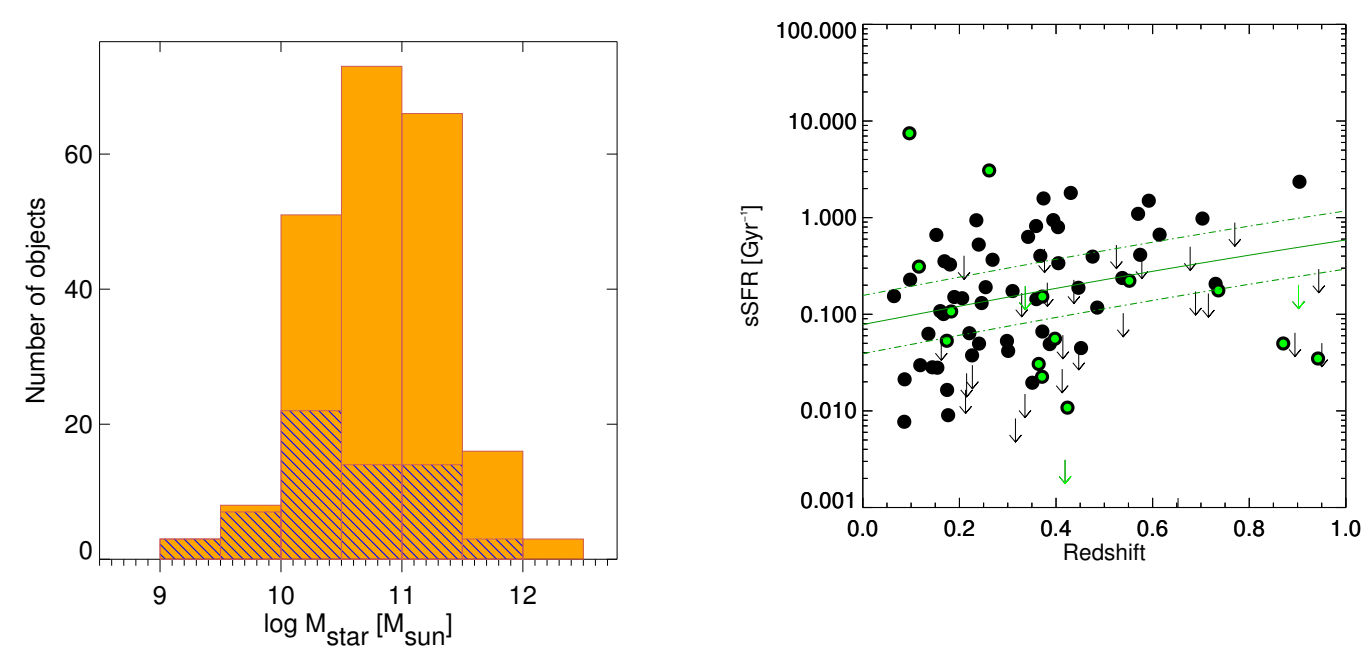

Fig. 14. Left panel: histogram of the logarithm of the stellar masses in $M_{\odot}$ derived for our sample of type 1 quasars. The hatched area represents uncertain values. Right panel: evolution of the main sequence SFR with redshift. The solid line represents the relation between sSFR and redshift and the two dashed lines are a factor of two above and below this line.
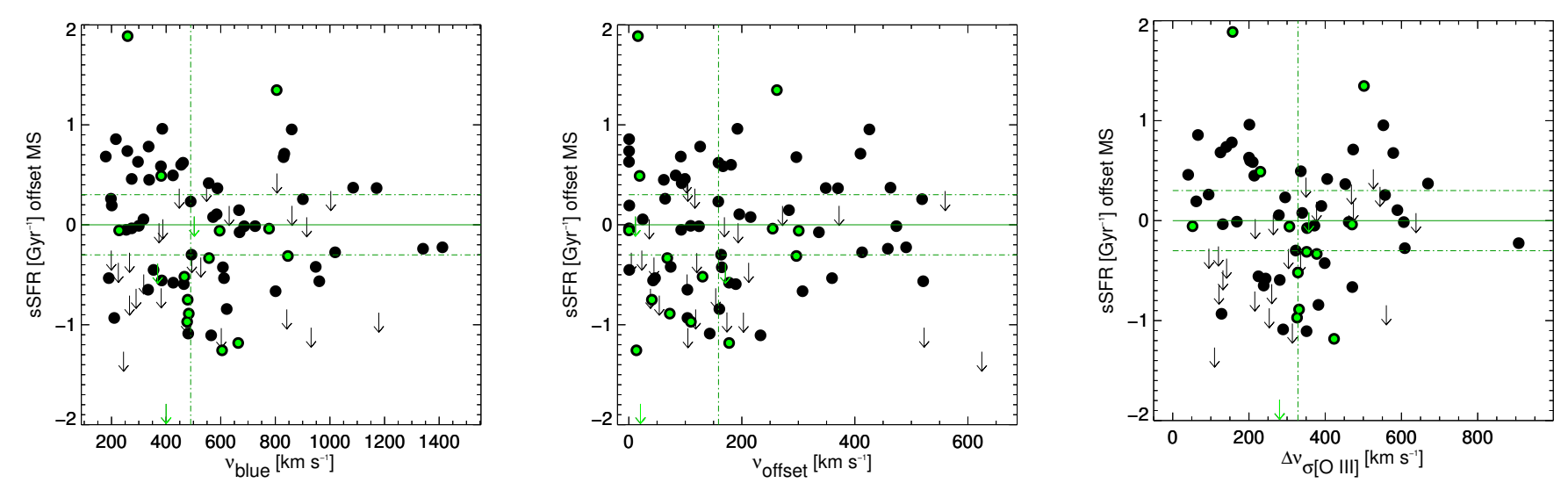

Fig. 15. sSFR offset from the main sequence versus the three emission line parameters characterizing the outflow: $v_{\text {blue }}, \Delta v_{\text {offset }}, \Delta v_{\sigma[0}$ III] . The green points represent the radio loud quasars.

In Fig. 14, right panel, we plot the sSFR versus redshift and we compare these measurements with the main sequence (adopting the Elbaz et al. 2011 formulation $^{7}$ ). The sSFR of the quasars

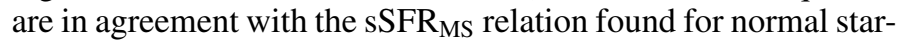
forming galaxies, although with a very large scatter that is due in part to the uncertainties on the stellar mass measurement. We investigate whether there is a relation between the position in this plane and the kinematic properties of the outflow. In Fig. 15, we compare the offset from the main sequence of $\mathrm{SF}\left(\mathrm{sSFR}_{\text {offset }}\right.$, a measure of the starburstiness of quasars) to the three kinematic parameters. Again, analogously to the SFR, we do not find a clear relation between these quantities and $\mathrm{SSFR}_{\text {offset }}$.

\section{Discussion}

\subsection{Evidence for a SB-outflow connection?}

The origin of the fast ionized gas outflows is controversial since in general it is not possible to establish unambiguously whether they are produced by the intense star formation, by the AGNs, or by a combination of the two processes. Galactic winds produced by outflows driven by thermal energy from supernova explosions or by stellar winds typically exhibit outflow velocities in the

\footnotetext{
$s S F R_{\mathrm{MS}}=26 \times t_{\text {cosmic }}^{-2.2}$ in $\left[\mathrm{Gyr}^{-1}\right]$ with $t_{\text {cosmic }} \sim \frac{28}{1+(1+z)^{2}}$ Gyr.
}

$100-500 \mathrm{~km} \mathrm{~s}^{-1}$ range (Rupke et al. 2005) and outflows with significantly higher velocities $\left(|v|>1000 \mathrm{~km} \mathrm{~s}^{-1}\right)$ are generally interpreted as driven by an AGN (e.g. Tremonti et al. 2007; Cicone et al. 2014). However, according to Diamond-Stanic et al. (2012) ram pressure from supernovae and stellar winds is sufficient to produce outflows with velocity higher than $1000 \mathrm{~km} \mathrm{~s}^{-1}$ without needing to invoke feedback from an active galactic nucleus.

Blue star-forming galaxies show a positive correlation between SFR and outflows. For example, Weiner et al. (2009) found that the outflow velocity scales linearly with SFR as $V_{\text {wind }} \sim S F R^{0.3}$ in blue star-forming galaxies at $z \sim 1.4$, similar to the scaling found by Martin (2005) in low-redshift ULIRGs. For quasars no obvious relation between the wind velocity and infrared luminosity has been discovered. For example, Veilleux et al. (2013) found that the velocity of the $\mathrm{OH}$ absorption line does not correlate with the star formation rate in a sample of ULIRGs and QSOs. A similar result is found by Rupke \& Veilleux (2013), considering both ionized and neutral gas outflow in six ULIRGs. These authors did not find any relation between the $\mathrm{OH}$ outflow velocities and the star formation. However, they find a non-linear relation between the velocity of the molecular outflow and the AGN luminosity, e.g. more blueshifted $\mathrm{OH}$ components are found in more AGN luminous sources. 
We have looked for a relationship between the SFR and the strength of the outflow, considering many different outflow parameters (e.g. $v_{\text {blue }}, \Delta v_{\text {offset }}, \Delta v_{\sigma[\mathrm{O} \text { III }]}$ ). We consider separately the two classes of SFd and AGNd quasars (Fig. 11, bottom panel), in which the driver mechanism of the outflow is probably different. No clear trend emerges between the star formation rate and the velocity of the outflow. However, we do not find any relation between the outflow and the properties of the AGNd quasars, as is expected if the outflow is ultimately driven by the radiative output of the quasar and as was found instead for molecular outflows in quasars by Veilleux et al. (2013). We cannot rule out that both AGN and stellar processes contribute to driving the outflow and weaken any relations, especially in quasars with a high star-forming activity. This is in agreement with what has been found by other previously cited authors, i.e. we do not find any obvious relation between the wind velocity and infrared luminosity in the quasar sample.

If we separate the quasars into two classes of quasars with weak and strong outflow, an interesting result emerges. We do not find that the mean star formation rate is lower in host galaxies that exhibit the strongest outflows, as predicted by the basic AGN negative feedback model; instead, it is consistent or even higher than in weak outflow quasars. However, it is worth noting some important shortcomings and uncertainties of this approach.

- There could be a significant delay between the starburst phase and the peak of nuclear optical AGN activity (e.g. Matsuoka et al. 2011; Davies et al. 2007; Bennert et al. 2008; Schawinski et al. 2009; Wild et al. 2010; Yesuf et al. 2014) estimated in the range of 100-400 Myr. The quasar phase is expected to last for a typical lifetime of 16-25 Myr (Gonçalves et al. 2008) consistent with the estimate of $>10^{7}$ yr by Jakobsen et al. 2003. The timescale for star formation, i.e. over which star formation remains constant, can vary by a few 100 Myr (e.g. de Grijs 2001). Therefore, a substantial time lag between the quasars phase and the quenching of the star formation rate in the host galaxy could wash out the most significant signatures of negative feedback. However, we note that if powerful winds are able to sweep away the interstellar dust, we would expect an underluminous infrared galaxy host, even if the star formation proceeds.

- Another possible interpretation of this result is that the AGN is stimulating the SFR as predicted by a positive feedback scenario. In this interpretation the AGN outflow triggers star formation in the gas-rich galaxies by overcompressing cold dense gas and thus providing positive feedback. AGNs with pronounced radio jets exhibit a much higher star formation rate than the purely X-ray selected ones (Zinn et al. 2013). In another case (Cresci et al. 2015), both types of feedback are observed in the same galaxy: the outflow removes the gas from the host galaxy (negative feedback), but also triggers star formation inducing pressure at the edges of the outflow (positive feedback). We expect to see the positive feedback effect especially in radio loud quasars. Interestingly, excluding radio loud quasars in our analysis, the difference between the mean SFR in the two groups decreases.

- The blueshifted wings of [O III] are typically seen only in the inner kpc of NLRs in local AGNs. So their detections imply outflows over regions that are much smaller than the starforming portion of typical host galaxies and therefore these outflows may not be able to quench the star formation rate. Integral field spectroscopic data are crucial to answering to this question.
- Since the Herschel spatial resolution does not allow us to measure the SFR in the nuclear region (on a subkilo parsec scale), we cannot rule out the possibility that the impact of negative feedback even in the most luminous systems only has an affect in the very central regions (below $100 \mathrm{pc}$ ), as suggested recently in the model of Roos et al. (2015).

- In this work we have considered the SFR, since the stellar mass in type 1 quasars is a difficult parameter to determine. It is possible that the sSFR correlates with the mass loading factor or the kinetic power of the outflow, not with the velocity. However, we cannot measure it with this data so we use the velocity as a proxy of the strength of the outflow.

Keeping in mind the discussed caveats, our results seem to disfavour the AGN negative feedback scenario, according to which AGN outflows on the QSO phase sweep out the gas from the host galaxy suppressing the star formation.

Our results are more in agreement with works that seems to demonstrate that AGNs do not regulate starbursts in the overall galaxy. For example Debuhr et al. (2010), found that SMBH feedback has little effect on the number of stars formed. In the theoretical model of Cen (2012) starburst and AGN growth are not coeval in this model and AGN activities are expected to outlive the starburst, in agreement with observations (e.g. Georgakakis et al. 2008). In a recent simulation Gabor \& Davé (2015) found that AGN feedback drives bursty, high velocity, hot outflows with mass rates peaking briefly near the SFR but that have little impact on the star-forming gas disk. The authors conclude that the star formation is not quenched by AGN feedback, and they suggested that the key physical element required to produce a galaxy red sequence is not expelling gas from galaxies, but rather preventing gas from accreting. Other processes than AGN negative feedback are able to transform a star-forming galaxy into a quiescent system, for example the socalled strangulation mechanism (e.g Larson et al. 1980; Balogh et al. 2000). In this case star formation in the host continues until the available gas is completely used up and the star formation is not suddenly quenched as in the negative feedback model. In a recent analysis Peng et al. (2015) found that strangulation is the primary mechanism acting in local galaxies.

The increased probability of an AGN being hosted by a starforming galaxy (e.g. Santini et al. 2012) may be a consequence of the relationship between gas content and AGN activity. If this is the case, the relationship between AGN bolometric luminosity and the SFR found by Netzer (2009) naturally arises. This relationship might ultimately be caused by secular mechanisms of gas inflow or merger mechanisms between galaxies. What emerges clearly is that the complex interplay between AGN outflows and star formation rate is far from being understood.

\section{Summary and conclusions}

The aim of this paper is to test the negative AGN feedback scenario in its simplest version according to which QSO driven outflows are able to suddenly quench the SFR in the host, clearing out or heating the interstellar medium of the galaxy. We have considered 132 quasars from the SDSS quasars observed in photometric mode by the infrared satellite Herschel at redshifts of less than 1 (to cover the [O III] line in the SDSS spectra) with high quality fits and spectra.

To investigate the presence of an outflow, we focus on the $[\mathrm{O} \mathrm{III}]$ and $[\mathrm{O} \mathrm{II}]$ emission line, in particular on three parameters (the maximum blue velocity, $v_{\text {blue }}=|v 10-v 50|$; the offset of the broad emission, $\Delta v_{\text {offset }}=|v 95+v 5| / 2-v 50$; and the 
$\sigma$ excess with respect to the $[\mathrm{O}$ II $]$ line, $\Delta v_{\sigma[\mathrm{O} \text { III }]}=\left(\sigma([\mathrm{O} \text { III }])^{2}-\right.$ $\left.\left.\sigma([\mathrm{OII}])^{2}\right)^{0.5}\right)$ assuming that a broad, blueshifted wing is a signature of an outflow.

Since the SFR evolves with redshift and it is dependent on the stellar mass of the galaxy (galaxies at higher redshift are typically more massive and more star forming), we consider the mean SFR in bins of redshift for strong and weak outflow galaxies. We do not see that strong outflow galaxies have a SFR lower than weak-outflow galaxies as was predicted by the negative AGN feedback scenario. Instead, we found that the SFRs in the two groups are comparable or even higher for the outflow dominated galaxies. We found an analogous result considering the offset from the main sequence of the sSFR. The stellar masses derived from SED fitting in type 1 quasars is uncertain because of the AGN contamination in IR. However, for the quasars with trustable measurements in which the stellar component dominates in the range $1-3 \mu \mathrm{m}$, we find that the redshift evolution of the specific SFR for the hosts in our sample of type 1 QSOs is in agreement with that observed for star-forming galaxies.

To investigate the main driver of the outflow, we divided our sample into two groups: the AGN-dominated quasars (defined as $\left.L_{\mathrm{SB}} / L_{\mathrm{tot}}<0.5\right)$ and the SF-dominated quasars $\left(L_{\mathrm{SB}} / L_{\mathrm{tot}}>\right.$ $0.5)$. We find that by focusing only on SF-dominated quasars we did not find a correlation between the SFR and the velocity, as found for starburst galaxies; rather, a slight positive trend was found. Instead in the AGN-dominated quasars do not find any clear relationship between the outflow velocities and the SFR or the AGN properties (Eddington ratio, [O III] luminosity, or total infrared luminosity).

We conclude that the negative feedback scenario is disfavoured by our results and that other possible mechanisms could be responsible for quenching star formation. In our large sample of quasars, a high level of SFR coexists with powerful outflow and, moreover, outflow dominated host galaxies have star formation rates consistent with or slightly larger than galaxies showing weak outflows. Different possibilities can explain the lack of observational evidence of negative feedback, including the possibility that AGN driven outflows are not the main mechanism by which the star formation in blue star-forming galaxies is suppressed.

Acknowledgements. We thank the referee for his/her accurate revision of the paper. B.B. acknowledges support from grant PRIN-INAF 2011 "Black hole growth and AGN feedback through the cosmic time". M.B. acknowledges support from the FP7 Career Integration Grant "eEASy": "supermasssive black holes through cosmic time: from current surveys to eROSITAEuclid Synergies" (CIG 321913). Funding for SDSS-III has been provided by the Alfred P. Sloan Foundation, the Participating Institutions, the National Science Foundation, and the US Department of Energy Office of Science. The SDSS-III web site is http://www.sdss3.org/. SDSS-III is managed by the Astrophysical Research Consortium for the Participating Institutions of the SDSS-III Collaboration including the University of Arizona, the Brazilian Participation Group, Brookhaven National Laboratory, Carnegie Mellon University, University of Florida, the French Participation Group, the German Participation Group, Harvard University, the Instituto de Astrofisica de Canarias, the Michigan State/Notre Dame/JINA Participation Group, Johns Hopkins University, Lawrence Berkeley National Laboratory, Max Planck Institute for Astrophysics, Max Planck Institute for Extraterrestrial Physics, New Mexico State University, New York University, Ohio State University, Pennsylvania State University, University of Portsmouth, Princeton University, the Spanish Participation Group, University of Tokyo, University of Utah, Vanderbilt University, University of Virginia, University of Washington, and Yale University.

\section{References}

Alexander, D. M., Swinbank, A. M., Smail, I., McDermid, R., \& Nesvadba, N. P. H. 2010, MNRAS, 402, 2211

Balogh, M. L., Navarro, J. F., \& Morris, S. L. 2000, ApJ, 540, 113
Bauer, A. E., Drory, N., Hill, G. J., \& Feulner, G. 2005, ApJ, 621, L89 Bennert, N., Canalizo, G., Jungwiert, B., et al. 2008, ApJ, 677, 846 Benson, A. J., Frenk, C. S., Baugh, C. M., Cole, S., \& Lacey, C. G. 2003, MNRAS, 343, 679

Bian, W., Yuan, Q., \& Zhao, Y. 2005, MNRAS, 364, 187

Bicknell, G. V., Sutherland, R. S., van Breugel, W. J. M., et al. 2000, ApJ, 540, 678

Bolzonella, M., Kovač, K., Pozzetti, L., et al. 2010, A\&A, 524, A76

Brusa, M., Bongiorno, A., Cresci, G., et al. 2015, MNRAS, 446, 2394 Bruzual, G., \& Charlot, S. 2003, MNRAS, 344, 1000

Cano-Díaz, M., Maiolino, R., Marconi, A., et al. 2012, A\&A, 537, L8

Cen, R. 2012, ApJ, 755, 28

Chabrier, G. 2003, PASP, 115, 763

Chary, R., \& Elbaz, D. 2001, ApJ, 556, 562

Cicone, C., Maiolino, R., Sturm, E., et al. 2014, A\&A, 562, A21

Ciotti, L., \& Ostriker, J. P. 2007, ApJ, 665, 1038

Cresci, G., Mainieri, V., Brusa, M., et al. 2015, ApJ, 799, 82

Croton, D. J. 2006, MNRAS, 369, 1808

Daddi, E., Dickinson, M., Morrison, G., et al. 2007, ApJ, 670, 156

Dale, D. A., Helou, G., Magdis, G. E., et al. 2014, ApJ, 784, 83

Davies, R. I., Müller Sánchez, F., Genzel, R., et al. 2007, ApJ, 671, 1388

de Grijs, R. 2001, Astron. Geophys., 42, 12

De Robertis, M. M., \& Osterbrock, D. E. 1984, ApJ, 286, 171

De Robertis, M. M., \& Shaw, R. A. 1990, ApJ, 348, 421

Debuhr, J., Quataert, E., Ma, C.-P., \& Hopkins, P. 2010, MNRAS, 406, L55

Di Matteo, T., Springel, V., \& Hernquist, L. 2005, Nature, 433, 604

Diamond-Stanic, A. M., Moustakas, J., Tremonti, C. A., et al. 2012, ApJ, 755, L26

Dimitrijević, M. S., Popović, L. Č., Kovačević, J., Dačić, M., \& Ilić, D. 2007, MNRAS, 374, 1181

Draine, B. T., \& Li, A. 2007, ApJ, 657, 810

Elbaz, D., Daddi, E., Le Borgne, D., et al. 2007, A\&A, 468, 33

Elbaz, D., Dickinson, M., Hwang, H. S., et al. 2011, A\&A, 533, A119

Fabian, A. C. 2012, ARA\&A, 50, 455

Ferrarese, L., \& Merritt, D. 2000, ApJ, 539, L9

Feruglio, C., Fiore, F., Carniani, S., et al. 2015, A\&A, 583, A99

Gabor, J. M., \& Davé, R. 2015, MNRAS, 447, 374

Gebhardt, K., Bender, R., Bower, G., et al. 2000, ApJ, 539, L13

Georgakakis, A., Nandra, K., Yan, R., et al. 2008, MNRAS, 385, 2049

Gonçalves, T. S., Steidel, C. C., \& Pettini, M. 2008, ApJ, 676, 816

Granato, G. L., De Zotti, G., Silva, L., Bressan, A., \& Danese, L. 2004, ApJ, 600,580

Greene, J. E., \& Ho, L. C. 2005, ApJ, 627, 721

Griffin, M. J., Abergel, A., Abreu, A., et al. 2010, A\&A, 518, L3

Hainline, L. J., Blain, A. W., Smail, I., et al. 2011, ApJ, 740, 96

Harrison, C. M., Alexander, D. M., Swinbank, A. M., et al. 2012, MNRAS, 426, 1073

Harrison, C. M., Alexander, D. M., Mullaney, J. R., \& Swinbank, A. M. 2014, MNRAS, 441, 3306

Heckman, T. M., Kauffmann, G., Brinchmann, J., et al. 2004, ApJ, 613, 109

Hopkins, P. F., Somerville, R. S., Hernquist, L., et al. 2006, ApJ, 652, 864

Hu, C., Wang, J.-M., Ho, L. C., et al. 2008, ApJ, 687, 78

Ishibashi, W., \& Fabian, A. C. 2012, MNRAS, 427, 2998

Jahnke, K., \& Macciò, A. V. 2011, ApJ, 734, 92

Jakobsen, P., Jansen, R. A., Wagner, S., \& Reimers, D. 2003, A\&A, 397, 891

Jiang, L., Fan, X., Ivezić, Ž., et al. 2007, ApJ, 656, 680

Kalfountzou, E., Jarvis, M. J., Bonfield, D. G., \& Hardcastle, M. J. 2012, MNRAS, 427, 2401

Kewley, L. J., Geller, M. J., \& Jansen, R. A. 2004, AJ, 127, 2002

Komossa, S., Xu, D., Zhou, H., Storchi-Bergmann, T., \& Binette, L. 2008, ApJ, 680,926

Kroupa, P. 2001, MNRAS, 322, 231

Larson, R. B., Tinsley, B. M., \& Caldwell, C. N. 1980, ApJ, 237, 692

Leipski, C., Meisenheimer, K., Walter, F., et al. 2014, ApJ, 785, 154

Mainieri, V., Bongiorno, A., Merloni, A., et al. 2011, A\&A, 535, A80

Maiolino, R., Gallerani, S., Neri, R., et al. 2012, MNRAS, 425, L66

Markwardt, C. B. 2009, in Astronomical Data Analysis Software and Systems XVIII, eds. D. A. Bohlender, D. Durand, \& P. Dowler, ASP Conf. Ser., 411, 251

Martig, M., Bournaud, F., Teyssier, R., \& Dekel, A. 2009, ApJ, 707, 250

Martin, C. L. 2005, ApJ, 621, 227

Matsuoka, K., Nagao, T., Marconi, A., Maiolino, R., \& Taniguchi, Y. 2011, A\&A, 527, A100

Meidt, S. E., Schinnerer, E., Knapen, J. H., et al. 2012, ApJ, 744, 17

Mullaney, J. R., Alexander, D. M., Fine, S., et al. 2013, MNRAS, 433, 622

Murphy, E. J., Condon, J. J., Schinnerer, E., et al. 2011, ApJ, 737, 67

Nagao, T., Murayama, T., \& Taniguchi, Y. 2001, ApJ, 549, 155

Nagao, T., Marconi, A., \& Maiolino, R. 2006, A\&A, 447, 157 
Nenkova, M., Sirocky, M. M. Ivezić, Ž., \& Elitzur, M. 2008, ApJ, 685, 147 Netzer, H. 2009, MNRAS, 399, 1907

Noeske, K. G., Weiner, B. J., Faber, S. M., et al. 2007, ApJ, 660, L43

Osterbrock, D. E. 1989, Astrophysics of gaseous nebulae and active galactic nuclei (Mill Valey, CA: University Science Books)

Pâris, I., Petitjean, P., Aubourg, É., et al. 2012, A\&A, 548, A66

Peng, Y., Maiolino, R., \& Cochrane, R. 2015, Nature, 521, 192

Perna, M., Brusa, M., Cresci, G., et al. 2015, A\&A, 574, A82

Poglitsch, A., Waelkens, C., Geis, N., et al. 2010, A\&A, 518, L2

Pozzetti, L., Bolzonella, M., Zucca, E., et al. 2010, A\&A, 523, A13

Pradhan, A. K., Montenegro, M., Nahar, S. N., \& Eissner, W. 2006, MNRAS, 366, L6

Richards, G. T., Fan, X., Newberg, H. J., et al. 2002, AJ, 123, 2945

Rieke, G. H., Alonso-Herrero, A., Weiner, B. J., et al. 2009, ApJ, 692, 556

Roche, P. F., Aitken, D. K., \& Smith, C. H. 1991, MNRAS, 252, 282

Roos, O., Juneau, S., Bournaud, F., \& Gabor, J. M. 2015, ApJ, 800, 19

Roseboom, I. G., Lawrence, A., Elvis, M., et al. 2013, MNRAS, 429, 1494

Rupke, D. S. N., \& Veilleux, S. 2013, ApJ, 768, 75

Rupke, D. S., Veilleux, S., \& Sanders, D. B. 2005, ApJ, 632, 751

Santini, P., Rosario, D. J., Shao, L., et al. 2012, A\&A, 540, A109

Schawinski, K., Virani, S., Simmons, B., et al. 2009, ApJ, 692, L19

Schlegel, D. J., Blanton, M., Eisenstein, D., et al. 2007, in AAS Meeting Abstracts, BAAS, 39, 132.29

Schneider, D. P., Richards, G. T., Hall, P. B., et al. 2010, AJ, 139, 2360

Seymour, N., Dwelly, T., Moss, D., et al. 2008, MNRAS, 386, 1695
Shao, L., Lutz, D., Nordon, R., et al. 2010, A\&A, 518, L26

Shen, Y., Richards, G. T., Strauss, M. A., et al. 2011, ApJS, 194, 45

Siebenmorgen, R., Krügel, E., \& Spoon, H. W. W. 2004, A\&A, 414, 123

Silva, L., Granato, G. L., Bressan, A., \& Danese, L. 1998, ApJ, 509, 103 Slone, O., \& Netzer, H. 2012, MNRAS, 426, 656

Stanley, F., Harrison, C. M., Alexander, D. M., et al. 2015, MNRAS, 453, 591

Tombesi, F., Sambruna, R. M., Reeves, J. N., et al. 2010, ApJ, 719, 700

Tombesi, F., Meléndez, M., Veilleux, S., et al. 2015, Nature, 519, 436

Tremonti, C. A., Moustakas, J., \& Diamond-Stanic, A. M. 2007, ApJ, 663, L77

Turnshek, D. A., Grillmair, C. J., Foltz, C. B., \& Weymann, R. J. 1988, ApJ, 325, 651

Veilleux, S. 1991, ApJ, 369, 331

Veilleux, S., Rupke, D. S. N., Kim, D.-C., et al. 2009, ApJS, 182, 628

Veilleux, S., Meléndez, M., Sturm, E., et al. 2013, ApJ, 776, 27

Véron-Cetty, M.-P., Joly, M., \& Véron, P. 2004, A\&A, 417, 515

Villar-Martín, M., Humphrey, A., Delgado, R. G., Colina, L., \& Arribas, S. 2011a, MNRAS, 418, 2032

Villar-Martín, M., Humphrey, A., Delgado, R. G., Colina, L., \& Arribas, S. 2011b, MNRAS, 418, 2032

Weiner, B. J., Coil, A. L., Prochaska, J. X., et al. 2009, ApJ, 692, 187

Whittle, M. 1985, MNRAS, 216, 817

Wild, V., Heckman, T., \& Charlot, S. 2010, MNRAS, 405, 933

Yesuf, H. M., Faber, S. M., Trump, J. R., et al. 2014, ApJ, 792, 84

Zakamska, N. L., \& Greene, J. E. 2014, MNRAS, 442, 784

Zinn, P.-C., Middelberg, E., Norris, R. P., \& Dettmar, R.-J. 2013, ApJ, 774, 66 


\section{Appendix A}

We explored the accuracy of our SED fitting code, since the best model that reproduces the photometric data points can potentially be subject to model degeneracy. To evaluate the uncertainties on the main quantities derived from the fit, i.e. $L_{\mathrm{AGN}}, L_{\mathrm{SF}}$, and $M_{\text {star }}$, in relation to the photometric uncertainties and model degeneracy, we performed some tests.

First of all, we built various theoretical SEDs, extracting photometric fluxes from our best fitting models. In each case the integrated luminosity of the SF and AGN component were known.

Second, we reproduced real cases, adding observed, realistic random errors to the theoretical photometric points (substituting randomly some photometric points with upper limits). Then we fit this SED obtaining $L_{\mathrm{SF}} L_{\mathrm{AGN}}$ observed. We repeated this procedure for 100 times.

In Fig. A.1, left panel, we show an example of a result of this analysis for one theoretical SED. We plotted the distribution of the values obtained in 100 iterations, and we compared the obtained values with the theoretical value shown with a dashed line. In each iteration the best model is composed of different templates; however, the distribution of the integrated luminosities are centred on the expected value. In Fig. A.1, right panel, we report in percentage the distribution of the relative errors for the main quantities $L_{\mathrm{AGN}}, L_{\mathrm{SF}}$, and $M_{\mathrm{star}}$ in all the iterations compared to the errors that we obtained in the theoretical case.

As a consistency check, we compare the colour obtained by Veilleux et al. (2009) for a sample of ultraluminous infrared galaxies and Palomar Green quasars with Spitzer. We derive the flux ratios at different wavelengths from the SEDs best fitting model. In Fig. A.2, left panel, we compare $F_{30 \mu \mathrm{m}} / F_{15 \mu \mathrm{m}}$ with respect to the flux ratio between 5 and $25 \mu \mathrm{m}$ (mid-IR) and between 40 and $120 \mu \mathrm{m}$ (far-IR). In the right panel, we plot $F_{15 \mu \mathrm{m}} / F_{6 \mu \mathrm{m}}$ with respect to $F_{30 \mu \mathrm{m}} / F_{6 \mu \mathrm{m}}$. In both cases our quasars extend the relation found by Veilleux et al. (2009) downward. 
B. Balmaverde et al.: Ionized gas outflow and star formation
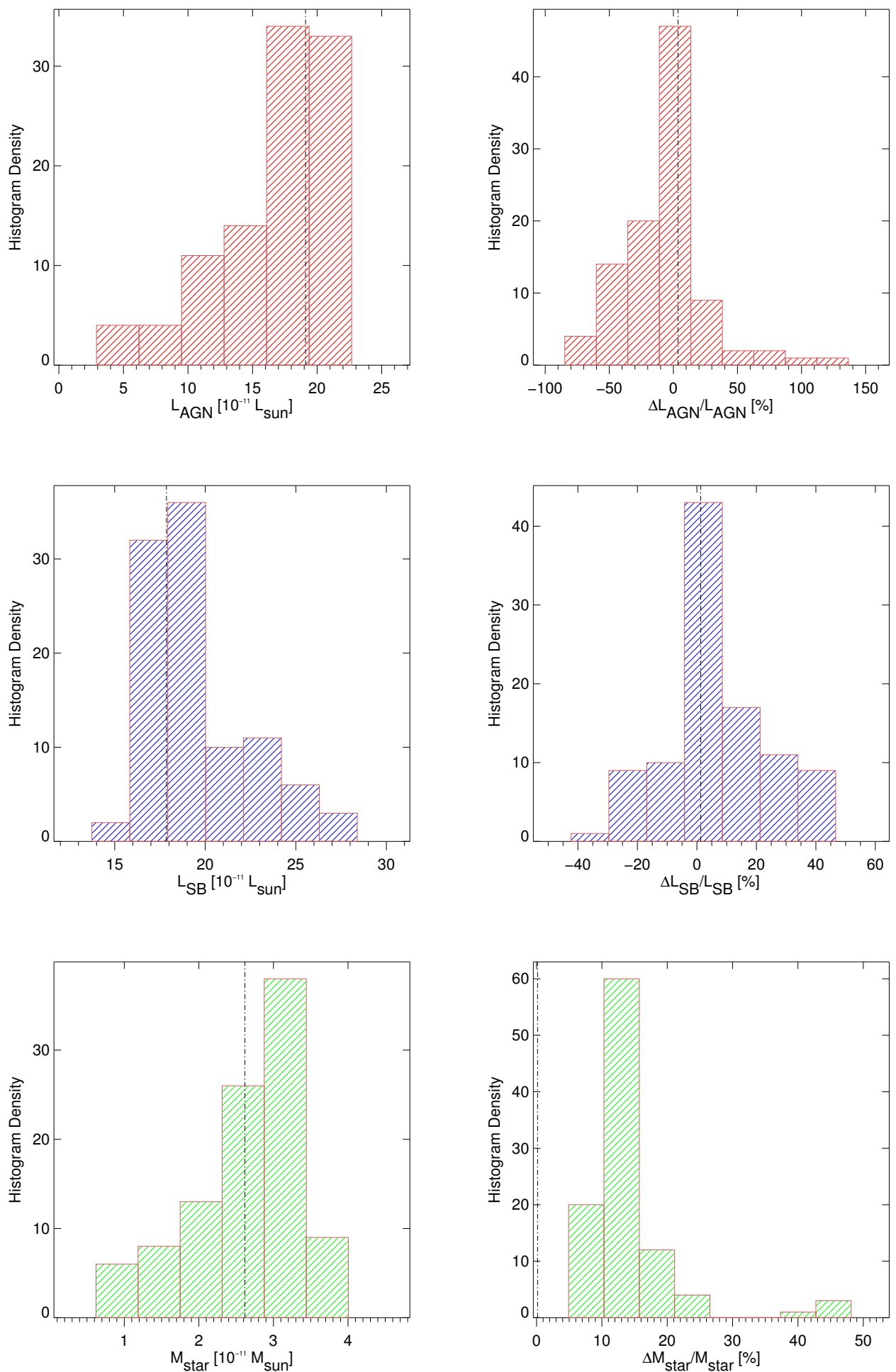

Fig. A.1. Example of a SED fitting test. Left panel: distribution of the AGN luminosity, SB luminosity, and stellar mass obtained in 100 iterations obtained from theoretical SEDs, and by varying the photometric points according to the observed uncertainties compared to the theoretical value represented by the dashed line. Right panel: as before, but we plot the distribution in percentage of the relative errors for the parameters in each iteration. 
A\&A 585, A148 (2016)
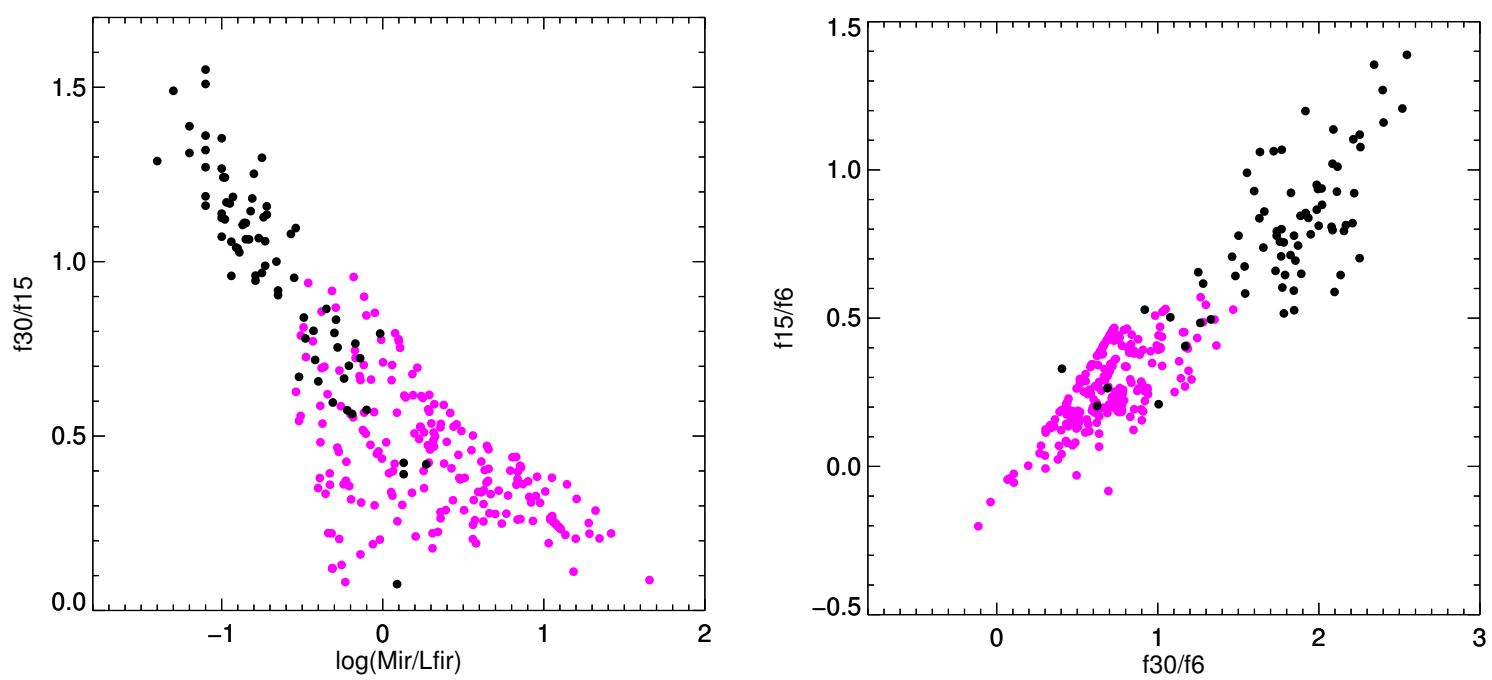

Fig. A.2. Comparison of SED colours derived from the SED with respect to a sample of PG quasars with Spitzer measurements and ULIR galaxies (black points, from Veilleux et al. 2009) and our sample of quasars (magenta points). 